\title{
Electronic Prescribing: An Examination of Cost Effectiveness, Clinician Adoption and Limitations
}

\author{
Annie Hahn ${ }^{1, *}$, Annesha Lovett ${ }^{2}$ \\ ${ }^{1}$ College of Pharmacy, Mercer University, Atlanta, 30341, Georgia, United States \\ ${ }^{2}$ College of Pharmacy Faculty, Mercer University, Atlanta, 30341, Georgia, United States \\ *Corresponding Author: annie.hahn@live.mercer.edu
}

Copyright $@ 2014$ Horizon Research Publishing All rights reserved.

\begin{abstract}
E-prescribing has flourished due to the promise of improving efficiency and decreasing prescription errors caused by its handwritten counterpart, yet only $44 \%$ of doctor's offices use paperless prescriptions. Many studies have assessed the value of e-prescribing in the clinical setting, yet there is no all-inclusive summation of these findings. The aim of this study was to review the literature within the last decade pertaining to the cost effectiveness, clinician adoption and limitations of e-prescribing. Journal articles from January 1, 2003 through May 1, 2013 were compiled through use of the search engines: PubMed, International Pharmaceutical Abstracts (IPA), and Google Scholar. A total of 56 peer-reviewed articles were included in this review. Trends show that most clinicians view e-prescribing positively despite some limitations. Limitations of e-prescribing include cost of implementation and maintenance, insufficient training, and lack of standardization. The cost to implement an integrated system with alerts and decision support is $\$ 29,000$ per physician for the first year and $\$ 4,000$ every year thereafter. Furthermore, e-prescribing has little disruption to workflow and no statistically significant increase in processing time. Although limitations exist, an expansion of e-prescribing is expected in the future. Efforts should be increased to promote clinicians adopting e-prescribing.
\end{abstract}

Keywords E-Prescribing, Pharmacy Informatics, Clinical Pharmacist, HITECH Act, and Computerized Provider Order Entry (CPOE)

\section{Introduction}

Electronic prescribing (or e-prescribing) is the electronic transmission of prescriptions or prescription-related information between a prescriber, dispenser, pharmacy benefit manager, or health plan [21].In the last 10 years, the use of e-prescribing has flourished due to the promise of improving efficiency and decreasing prescription errors caused by its handwritten counterpart, yet according to 2012 estimates, only $44 \%$ of doctor's offices use paperless prescriptions $[1,2,3]$. However, this is a drastic increase from $12 \%$ utilization in 2009. While there was initial opposition towards e-prescribing due to perceptions of increased costs, training requirements, and time constraints, many studies have been published to address those concerns.For example, many clinicians felt that the use of e-prescribing would disrupt workflow and take more time.However, it has been shown that e-prescribing actually saves time and can be implemented into the workflow with proper training [4, 5].The purpose of this review was to discuss e-prescribing as a preferred form of prescribing and its current impact.With the Medicare Modernization Act of 2003 pushing for e-prescribing and many other insurers following suit, this article summarizes the present state of e-prescribing and its future utilization [6].In addition, the importance of clinician acceptance of electronic prescribing and methods to improve integration are discussed.Clinical pharmacists have an opportunity to detect prescribing errors and improve prescription quality through the use of electronic prescribing.Furthermore, electronic prescribing has been used to monitor and assess patient compliance and prescription discrepancies [23].Several studies show that electronic prescribing has been shown to reduce prescribing errors in United States hospitals [49,50,51,52,53,54]. However, there is no consensus on best practices in the use of electronic prescribing.Further research is needed to explore implementation of electronic prescribing within hospital systems considering that only $67 \%$ of eligible users were utilizing electronic health records (EHR) in 2012, and an even lower percentage for electronic prescriptions [7].With more prescribers utilizing electronic prescribing and pharmacists processing electronic prescriptions over the next few years, more analysis of e-prescribing methods can be made and best practices can be developed.These assessments can help patients reach disease state goals and help clinicians reach the overall goal to enhance patient care through better integration and communication between the patient, pharmacist and physician $[18,23,24]$. 


\section{Materials and Methods}

Journal articles were compiled through use of the following search engines: PubMed, International Pharmaceutical Abstracts (IPA), and Google Scholar. The key terms utilized were: 'future AND e-prescribing', 'future AND pharmacy informatics', 'e-prescribing AND limitations', 'clinical pharmacist AND e-prescribing', 'HITECH act AND electronic prescribing' and 'computerized provider order entry (CPOE).'

Articles selected for review were published from January 1, 2003 through May 1, 2013 (Table 1, Table 2). The articles required information on e-prescribing; particularly, articles pertaining to cost-effectiveness, limitations, clinician perception and future needs. Articles pertaining to pharmacy or the use of pharmacy informatics were also utilized as a secondary search and mainly focused on U.S. data. However, international studies were utilized to characterize crucial points or opinions about e-prescribing. Subsequent related U.S. articles were then searched to support those claims.
Articles were excluded from the search if they were written in or prior to 2002. Articles that concentrated on the utilization of electronic health records were also excluded as the primary objective was to review e-prescribing. An exception was two studies on public attitudes on electronic health records and studies that discussed the e-prescribing process. Finally, cost-effectiveness studies completed internationally were excluded since the values reported in the studies could not be accurately extrapolated to U.S. dollars. Once articles were retrieved, they were evaluated based on the Oxford Centre for Evidence-Based Medicine (Table 1). Next, they were summarized into tables based on subtopic (cost effectiveness, clinician adoption, and limitations), which were further organized by noting the year published, author(s), title, study design, intervention, findings, source for each study, and study grade (Table $2,3,4)$. The articles were assessed for quality by a second author to ensure that all articles met the inclusion criteria.

Table 1. Level of evidence for rating studies and grading recommendations, as per the Oxford Centre for Evidence-based Medicine (OCEBM)

\begin{tabular}{|l|l|}
\hline Level & \multicolumn{1}{|c|}{ Type of evidence } \\
\hline $1 \mathrm{a}$ & Systematic review with homogeneity of randomized control trials \\
\hline 1b & Individual randomized control trial with a narrow confidence interval \\
\hline $1 \mathrm{c}$ & All or none related outcome \\
\hline $2 \mathrm{a}$ & Systematic review with homogeneity of cohort studies \\
\hline 2b & Individual cohort study (including low-quality randomized control trials, e.g. less than 80\% follow-up) \\
\hline $2 \mathrm{c}$ & "Outcomes" Research; Ecological studies \\
\hline $3 \mathrm{a}$ & Systematic review with homogeneity of case-control studies \\
\hline $3 \mathrm{~b}$ & Individual case-control study \\
\hline 4 & Case-series (and poor-quality cohort and case-control studies) \\
\hline 5 & Expert opinion without explicit critical appraisal, or based on physiology, bench research or “first principles" \\
\hline & \\
\hline A & Consistent with level 1 studies \\
\hline B & Consistent level 2 or 3 studies or extrapolations from level 1 studies \\
\hline C & Level 4 studies or extrapolations from level 2 or 3 studies \\
\hline D & Level 5 evidence or troublingly inconsistent or inconclusive studies of any level \\
\hline &
\end{tabular}


Table 2. Summary of Articles:Cost effectiveness

\begin{tabular}{|c|c|c|c|c|c|c|c|}
\hline Year & Author & Title & Design & Intervention & Findings & Source & $\begin{array}{l}\text { Study } \\
\text { Grade }\end{array}$ \\
\hline 2003 & $\begin{array}{l}\text { Bartlett, Tamblyn, } \\
\text { Huang, Kawasumi, } \\
\text { Petrella, Dufour }\end{array}$ & $\begin{array}{l}\text { Evaluation of Standardized } \\
\text { Tasks for Primary Care } \\
\text { Physicians Using the MOXXI } \\
\text { Electronic Prescribing and } \\
\text { Integrated Drug Management } \\
\text { System }\end{array}$ & $\begin{array}{l}\text { Prospective } \\
\text { t-test }\end{array}$ & $\begin{array}{l}\text { Administering and } \\
\text { timing the completion of } \\
\text { a series of tasks by a } \\
\text { physician after a } 2 \text { week } \\
\text { training program }\end{array}$ & $\begin{array}{l}\text { - Physicians were given a } \\
\text { training session with the } \\
\text { e-prescribing software using } \\
\text { mock patients. } \\
\text { - They were then evaluated on } \\
\text { how quickly and efficiently they } \\
\text { were able to redo those tasks on } \\
\text { their own. } \\
\text { - Physicians were able to quickly } \\
\text { complete all tasks but only had } \\
\text { difficulty with changing the status } \\
\text { of a problem or with renewing } \\
\text { multiple prescriptions at once }\end{array}$ & $\begin{array}{c}\text { AMIA Annual } \\
\text { Symposium Archive }\end{array}$ & A \\
\hline 2003 & Corley & $\begin{array}{l}\text { Electronic prescribing: a } \\
\text { review of costs and benefits }\end{array}$ & Analysis & None & $\begin{array}{l}\text { - Improved physician, nurse and } \\
\text { staff efficiency calculated using } \\
\text { time trial comparisons between } \\
\text { paper and e-prescribing } \\
\text { - E-prescribing software is } \\
\text { cost-effective for all size } \\
\text { practices with a more rapid ROI } \\
\text { in larger practices }\end{array}$ & $\begin{array}{l}\text { Topics in Health } \\
\text { Information } \\
\text { Management }\end{array}$ & B \\
\hline 2003 & $\begin{array}{l}\text { Fischer, Stewart, } \\
\text { Mehta, Wax, Lapinsky }\end{array}$ & $\begin{array}{l}\text { Handheld Computing in } \\
\text { Medicine }\end{array}$ & $\begin{array}{l}\text { Systematic review } \\
\text { of literature }\end{array}$ & $\begin{array}{l}\text { Summary of articles and } \\
\text { current literature to } \\
\text { provide evidence-based } \\
\text { information }\end{array}$ & $\begin{array}{l}\bullet \text { Enough information was } \\
\text { collected to create an article to } \\
\text { increase awareness amongst } \\
\text { physicians about the potential } \\
\text { roles for portable devices and to } \\
\text { call for further evaluation for the } \\
\text { benefit of use }\end{array}$ & $\begin{array}{l}\text { Journal of American } \\
\text { Medical Informatics } \\
\text { Association }\end{array}$ & B \\
\hline 2004 & Tanne & $\begin{array}{l}\text { Electronic prescribing could } \\
\text { save at least } \$ 29 \text { billion }\end{array}$ & $\begin{array}{l}\text { Analysis } \\
\text { article }\end{array}$ & None & $\begin{array}{l}\text { - Assessment of physicians } \\
\text { sending eRx to pharmacies } \\
\text { - Cost in savings from preventing } \\
\text { duplications from multiple } \\
\text { doctors } \\
\text { - Cheaper prescription options } \\
\text { and alert about interactions and } \\
\text { incorrect dosages with clinical } \\
\text { decision support } \\
\text { - Downstream savings of } \$ 2 \\
\text { billion from preventing errors that } \\
\text { would result in doctor visits or } \\
\text { hospital stays }\end{array}$ & British Medical Journal & B \\
\hline 2005 & $\begin{array}{l}\text { Pizzi, Suh, Barone and } \\
\text { Nash }\end{array}$ & $\begin{array}{c}\text { Factors related to physicians' } \\
\text { adoption of electronic }\end{array}$ & Quantitative study & Electronic survey & $\begin{array}{l}\bullet 1,104 \text { responses recorded } \\
\bullet 19 \% \text { of responses from }\end{array}$ & $\begin{array}{l}\text { American Journal of } \\
\text { Medical Quality }\end{array}$ & A \\
\hline
\end{tabular}




\begin{tabular}{|c|c|c|c|c|c|c|c|}
\hline & & $\begin{array}{c}\text { prescribing: results from a } \\
\text { national survey }\end{array}$ & & & $\begin{array}{c}\text { physicians who used } \\
\text { e-prescribing } \\
\text { - Adoption can be stimulated by } \\
\text { improvements in the technology } \\
\text { and an organizational } \\
\text { commitment }\end{array}$ & & \\
\hline 2007 & $\begin{array}{l}\text { Donyai, O’Grady, } \\
\text { Jacklin, Barber, } \\
\text { Franklin }\end{array}$ & $\begin{array}{l}\text { The effects of electronic } \\
\text { prescribing on the quality of } \\
\text { prescribing }\end{array}$ & $\begin{array}{l}\text { Prospective } \\
\text { intervention } \\
\text { analysis }\end{array}$ & $\begin{array}{l}\text { Recording of pharmacy } \\
\text { interventions during a } 4 \\
\text { week period before and } \\
\text { after implementation of } \\
\text { electronic prescribing }\end{array}$ & $\begin{array}{c}\bullet \text { UK study } \\
\bullet \text { E-prescribing reduced } \\
\text { pharmacist intervention }(73 \rightarrow 45) \\
\text { as well as prescribing errors }(94 \\
\rightarrow 48) \\
\text { - Pharmacy needs to develop } \\
\text { ways to complement use of } \\
\text { electronic prescribing rather than } \\
\text { duplicate the benefits of it }\end{array}$ & $\begin{array}{c}\text { British Journal of } \\
\text { Clinical Pharmacology }\end{array}$ & B \\
\hline 2007 & Foxhall & $\begin{array}{l}\text { E-prescribing goes to } \\
\text { Washington. With the federal } \\
\text { government behind it, } \\
\text { electronic prescribing is } \\
\text { gaining speed, weight, and } \\
\text { more standards }\end{array}$ & Report & None & $\begin{array}{l}\text { - Medicare incentives are } \\
\text { pushing for e-prescribing } \\
\text { - In } 2013 \text {, there will be a } 1.5 \% \\
\text { Medicare Part B payment } \\
\text { adjustment to physicians if } \\
\text { e-prescribing is not utilized. } \\
\text { - In 2014, that adjustment goes } \\
\text { up to } 2 \% \text {. }\end{array}$ & Healthcare informatics & $\mathrm{D}$ \\
\hline 2007 & Gerstle, Lehmann & $\begin{array}{c}\text { Electronic prescribing } \\
\text { systems in pediatrics: the } \\
\text { rationale and functionality } \\
\text { requirements }\end{array}$ & $\begin{array}{l}\text { Technical } \\
\text { report }\end{array}$ & $\begin{array}{l}\text { Report discusses use of } \\
\text { e-prescribing in } \\
\text { pediatrics }\end{array}$ & $\begin{array}{l}\text { - Evaluates e-prescribing in all } \\
\text { aspects (from costs to technical } \\
\text { issues) }\end{array}$ & Pediatrics & B \\
\hline 2007 & $\begin{array}{l}\text { Grossman, Gerland, } \\
\text { Reed and Fahlman }\end{array}$ & $\begin{array}{c}\text { Physician's experiences using } \\
\text { commercial e-prescribing } \\
\text { systems }\end{array}$ & Discussion & $\begin{array}{l}44 \text { discussions from } \\
\text { respondents in } \\
\text { two-person teams in } 26 \\
\text { organizations via } \\
\text { telephone }\end{array}$ & $\begin{array}{l}\text { - 2/3 of practices used EMR } \\
\text { (integrated system) } \\
\text { - 1/3 stand-alone system } \\
\text { - Issue with maintaining patient } \\
\text { medication lists } \\
\text { - Lack of decision support } \\
\text { - Difficulty obtaining accurate } \\
\text { patient-specific formulary } \\
\text { information } \\
\text { - Limited connectivity with } \\
\text { pharmacies and mail-order PBMs } \\
\text { - Not enough staff to maintain } \\
\text { and support IT after } \\
\text { implementation } \\
\text { - All respondents believe } \\
\text { e-prescribing improves safety and } \\
\text { quality while increasing practice } \\
\text { efficiency }\end{array}$ & Health Affairs & B \\
\hline 2007 & Hollingworth, Devine, & The Impact of e-prescribing & Cross-sectional & Time-motion methods to & •No statistically significant & Journal of American & B \\
\hline
\end{tabular}




\begin{tabular}{|c|c|c|c|c|c|c|c|}
\hline & $\begin{array}{c}\text { Hansen, Lawless, } \\
\text { Comstock, } \\
\text { Wilson-Norton, Tharp, } \\
\text { Sullivan }\end{array}$ & $\begin{array}{c}\text { on Prescriber and Staff Time } \\
\text { in Ambulatory Care Clinics: } \\
\text { A Time-Motion Study }\end{array}$ & comparison study & $\begin{array}{l}\text { track staff and prescriber } \\
\text { tasks while using an } \\
\text { integrated system }\end{array}$ & $\begin{array}{l}\text { increase in time taken to } \\
\text { e-prescribe versus handwrite } \\
\text { prescriptions. } \bullet \text { E-prescribing has } \\
\text { no disruption to workflow and } \\
\text { therefore that reason can't be seen } \\
\text { as a barrier to adopting } \\
\text { e-prescribing practices. }\end{array}$ & $\begin{array}{c}\text { Medical Informatics } \\
\text { Association }\end{array}$ & \\
\hline 2007 & Scalise & The case for e-prescribing & Analysis & None & $\begin{array}{l}\text { - Lost productivity due to time } \\
\text { required to print PDA generated } \\
\text { prescriptions and fax pharmacies } \\
\text { (applies to controlled } \\
\text { medications) } \\
\text { - Program functionality } \\
\text { confusion } \\
\text { - Cost - software/hardware cost } \\
\text { ranges from } \$ 1,500 \text { - } \\
\$ 4,000 / \text { physician } \\
\text { - Advanced systems with } \\
\text { complex alerts and reminders are } \\
\$ 29,000 / \text { physician in the first year } \\
\text { and } \$ 4,000 \text { annually thereafter } \\
\text { - Waiting for an all-in-one } \\
\text { solution before investing in a } \\
\text { product } \\
\end{array}$ & $\begin{array}{l}\text { Hospital and Health } \\
\text { Networks }\end{array}$ & $\mathrm{B}$ \\
\hline 2008 & $\begin{array}{c}\text { Devine, } \\
\text { Wilson-Norton, } \\
\text { Lawless, Hansen, } \\
\text { Hollingworth, Fisk, } \\
\text { Sullivan }\end{array}$ & $\begin{array}{l}\text { Implementing an Ambulatory } \\
\text { e-Prescribing System: } \\
\text { Strategies Employed and } \\
\text { Lessons Learned to Minimize } \\
\text { Unintended Consequences }\end{array}$ & Analysis & None & $\begin{array}{l}\text { - CPOE improves quality of care } \\
\text { and reduces med errors } \\
\text { - Need an integrated, } \\
\text { community-based system for } \\
\text { adoption } \\
\text { - Need for ongoing training } \\
\text { - Key is team-oriented culture } \\
\text { (bridge between all points of } \\
\text { healthcare) }\end{array}$ & $\begin{array}{l}\text { Advances in Patient } \\
\text { Safety: Alternative } \\
\text { Approaches (Volume } \\
\text { 4: Technology and } \\
\text { Medication Safety) }\end{array}$ & $\mathrm{B}$ \\
\hline 2008 & $\begin{array}{c}\text { Fischer, Vogeli, } \\
\text { Stedman, Ferris, } \\
\text { Brookhart, Weissman }\end{array}$ & $\begin{array}{l}\text { Effect of electronic } \\
\text { prescribing with formulary } \\
\text { decision support on } \\
\text { medication use and cost }\end{array}$ & $\begin{array}{c}\text { Pre-post } \\
\text { study with } \\
\text { concurrent controls }\end{array}$ & $\begin{array}{c}\text { Analysis of } \\
\text { administrative data in } 2 \\
\text { large Massachusetts } \\
\text { insurers }\end{array}$ & $\begin{array}{c}\text { - Reduced spending on } \\
\text { medications due to formulary } \\
\text { decision support } \\
\text { - Physicians more likely to } \\
\text { prescribe tier } 1 \text { drugs } \\
\text { - } \$ 845,000 / 100,000 \text { patients } \\
\text { saving } \\
\end{array}$ & $\begin{array}{l}\text { Archives of Internal } \\
\text { Medicine }\end{array}$ & A \\
\hline 2008 & Rupp, Warholak & $\begin{array}{l}\text { Evaluation of e-prescribing in } \\
\text { chain community pharmacy } \\
\text { practice recommendations }\end{array}$ & $\begin{array}{l}\text { Descriptive, } \\
\text { nonexperimental, } \\
\text { cross-sectional } \\
\text { study }\end{array}$ & $\begin{array}{l}\text { Self-administered survey } \\
\text { and interviews of key } \\
\text { pharmacy operations and } \\
\text { information technology } \\
\text { management }\end{array}$ & $\begin{array}{c}\bullet 1,094 \text { surveys returned. } \\
\bullet \text { E-prescriptions were preferred } \\
\text { in each of the } 7 \text { desired outcomes } \\
\text { of care. } \\
\bullet \text { Benefits: legibility, improved } \\
\text { speed on efficiency of processing }\end{array}$ & $\begin{array}{c}\text { Journal of the } \\
\text { American Pharmacists } \\
\text { Association }\end{array}$ & $\mathrm{B}$ \\
\hline
\end{tabular}




\begin{tabular}{|c|c|c|c|c|c|c|c|}
\hline & & & & & $\begin{array}{c}\text { Disadvantage: prescribing errors } \\
\text { due to lack of training }\end{array}$ & & \\
\hline 2009 & $\begin{array}{c}\text { Balfour, Evans, } \\
\text { Januska, Lee, Lewis, } \\
\text { Nolan, Noga, Stemple, } \\
\text { Thapar }\end{array}$ & $\begin{array}{c}\text { Health Information } \\
\text { Technology - Results from a } \\
\text { Roundtable Discussion }\end{array}$ & Discussion & None & $\begin{array}{l}\text { - Challenges to adoption of } \\
\text { e-prescribing are high costs, } \\
\text { resistance to change, misaligned } \\
\text { incentives and fractured payment } \\
\text { system } \\
\text { - Benefits: avoid costs from } \\
\text { adverse drug events } \\
\text { - Impact on cost exceed a saving } \\
\text { of } \$ 800 \text { million over a } 5 \text { year } \\
\text { period by prescribers, dispenses } \\
\text { and beneficiaries } \\
\text { - E-rx transactions have risen } 3 x \\
\text { from } 2007 \text { to } 2008 \text { with over } 100 \\
\text { million transactions (started with } \\
700,000 \text { in 2004) }\end{array}$ & $\mathrm{JMCP}$ & $\mathrm{C}$ \\
\hline 2009 & Smith, Dang, Lee & $\begin{array}{c}\text { E-prescribing: Clinical } \\
\text { Implications for Patients with } \\
\text { Diabetes }\end{array}$ & Analysis & None & $\begin{array}{l}\text { - Mentions movement and } \\
\text { utilization of e-prescribing to help } \\
\text { pharmacy with reduction of errors } \\
\text { and clinical decision support } \\
\text { - Help reaching diabetes goals } \\
\text { - Indications of drug interactions } \\
\text { and importance of complete } \\
\text { medication profile } \\
\text { - Cases to prove potential } \\
\text { adverse interactions or incorrect } \\
\text { dispensing }\end{array}$ & $\begin{array}{c}\text { Journal of Diabetes } \\
\text { Science and } \\
\text { Technology }\end{array}$ & $\mathrm{C}$ \\
\hline 2010 & Brooks, Sonnenschien & $\begin{array}{l}\text { E-prescribing: where health } \\
\text { information and patient care } \\
\text { intersect }\end{array}$ & Analysis & None & $\begin{array}{l}\text { - MTM can help reduce rising } \\
\text { costs of healthcare } \\
\text { - E-prescribing allows for most } \\
\text { medically appropriate and cost } \\
\text { effective prescription at point of } \\
\text { care and transmitting to patient's } \\
\text { pharmacy of choice } \\
\text { - Makes process more efficient }\end{array}$ & $\begin{array}{l}\text { Journal of Healthcare } \\
\text { Information } \\
\text { Management }\end{array}$ & $\mathrm{D}$ \\
\hline 2010 & $\begin{array}{l}\text { Kaushal, Kern, } \\
\text { Barron, Quaresimo, } \\
\text { Abramson }\end{array}$ & $\begin{array}{c}\text { Electronic Prescribing } \\
\text { Improves Medication Safety } \\
\text { in Community-Based Office } \\
\text { Practices }\end{array}$ & $\begin{array}{l}\text { Prospective, } \\
\text { non-randomized } \\
\text { study }\end{array}$ & $\begin{array}{c}\text { Use of commercial, } \\
\text { stand-alone e-prescribing } \\
\text { system with clinical } \\
\text { decision support } \\
\text { including dosing } \\
\text { recommendations and } \\
\text { checks for drug-allergy } \\
\text { interactions, drug-drug } \\
\text { interactions and } \\
\text { duplicate therapies } \\
\end{array}$ & $\begin{array}{l}\text {-Error rates when changed to } \\
\text { e-prescriptions after a year } \\
\text { follow-up showed a sevenfold } \\
\text { decrease in error rates compared } \\
\text { to those who stayed with paper } \\
\text { prescriptions. }\end{array}$ & $\begin{array}{l}\text { Journal of General } \\
\text { Internal Medicine }\end{array}$ & B \\
\hline
\end{tabular}




\begin{tabular}{|c|c|c|c|c|c|c|c|}
\hline 2011 & Kannry & $\begin{array}{l}\text { Effect of e-prescribing } \\
\text { systems on patient safety }\end{array}$ & Overview study & None & $\begin{array}{l}\text { - Limited evidence suggests that } \\
\text { e-prescribing with medical } \\
\text { decision support can improve } \\
\text { patient safety } \\
\text { - Medicare and Medicaid } \\
\text { incentives to push for } \\
\text { e-prescribing } \\
\text { - E-prescribing as its own source } \\
\text { of medication error }\end{array}$ & $\begin{array}{l}\text { The Mount Sinai } \\
\text { Journal of Medicine }\end{array}$ & $\mathrm{C}$ \\
\hline 2011 & Liu, Burkhart, Bell & $\begin{array}{l}\text { Evaluation of the NCPDP } \\
\text { Structured and Codified Sig } \\
\text { Format for e-prescriptions }\end{array}$ & $\begin{array}{l}\text { Retrospective } \\
\text { cross-sectional } \\
\text { study }\end{array}$ & $\begin{array}{l}\text { Evaluation of } \\
\text { ambulatory } \\
\text { e-prescriptions based on } \\
\text { the actual words used by } \\
\text { prescribers in the sig } \\
\text { field to the equivalent } \\
\text { terms in the designated } \\
\text { terminology } \\
\end{array}$ & $\begin{array}{l}\bullet 95 \% \text { of sigs were correctly } \\
\text { represented } \\
\text { - Some ambiguities - but mainly } \\
\text { from multi-step instructions } \\
\text { - Most errors were administration } \\
\text { timings and dose delivery } \\
\text { methods }\end{array}$ & $\begin{array}{l}\text { Journal of the } \\
\text { American Medical } \\
\text { Informatics } \\
\text { Association }\end{array}$ & B \\
\hline 2011 & $\begin{array}{l}\text { Michelis, Hassouna, } \\
\text { Owlia, Kelahan, } \\
\text { Young, Choi }\end{array}$ & $\begin{array}{l}\text { Effect of electronic } \\
\text { prescription on attainment of } \\
\text { cholesterol goals }\end{array}$ & $\begin{array}{l}\text { Retrospective } \\
\text { cohort study }\end{array}$ & $\begin{array}{l}\text { Query of } 796 \text { patients } \\
\text { not at LDL goal at } \\
\text { baseline }\end{array}$ & $\begin{array}{l}\text { - Patients receiving an eRX were } \\
59 \% \text { more likely to achieve LDL } \\
\text { goal } \\
\text { - Reasons may be due to } \\
\text { formulary decision support and } \\
\text { lower cost of medications } \\
\text { - For every } \$ 10 \text { increase in } \\
\text { prescription price, the likelihood } \\
\text { of being at goal decreased by } 5 \%\end{array}$ & Clinical Cardiology & B \\
\hline 2012 & $\begin{array}{c}\text { Gilligan, Miller, } \\
\text { Mohney, Montenegro, } \\
\text { Schwarz, Warholak }\end{array}$ & $\begin{array}{c}\text { Analysis of pharmacists' } \\
\text { interventions on electronic } \\
\text { versus traditional } \\
\text { prescriptions in } 2 \text { community } \\
\text { pharmacies }\end{array}$ & $\begin{array}{c}\text { Observational } \\
\text { prospective design }\end{array}$ & $\begin{array}{c}\text { Collect and examine data } \\
\text { from } 2 \text { community chain } \\
\text { pharmacies }\end{array}$ & $\begin{array}{l}\text { - } 1,678 \text { new prescriptions were } \\
\text { reviewed and } 153 \text { needed } \\
\text { intervention over a } 13 \text { day period } \\
\text { - } 11 \text { hours and } 58 \text { minutes were } \\
\text { spent on the interventions } \\
\text { - Reasons for intervention: } \\
\text { excessive quantity/duration and } \\
\text { violating legal requirements } \\
\text { - Difference in intervention rate } \\
\text { between e-prescribing and } \\
\text { handwritten prescriptions were } \\
\text { not statistically significant }\end{array}$ & $\begin{array}{l}\text { Research in Social and } \\
\text { Administrative } \\
\text { Pharmacy }\end{array}$ & A \\
\hline 2012 & McBride & E-prescribing prevents errors & Analysis & None & $\begin{array}{c}\bullet 67 \% \text { of physicians are } \\
\text { e-prescribing } \\
\text { - Point of e-prescribing is to give } \\
\text { more data to have better safety } \\
\text { checks and not save time } \\
\bullet \text { Vital communication link } \\
\text { between physician, pharmacists } \\
\text { and patients }\end{array}$ & Medical Economics & $\mathrm{C}$ \\
\hline
\end{tabular}




\begin{tabular}{|c|c|c|c|c|c|c|c|}
\hline 2012 & Surescripts & $\begin{array}{l}\text { E-prescribing shown to } \\
\text { improve outcomes, save } \\
\text { billions }\end{array}$ & $\begin{array}{c}\text { Post-hoc } \\
\text { quantitative study }\end{array}$ & $\begin{array}{l}\text { Collaboration between } \\
\text { Surescripts, pharmacies, } \\
\text { and PBMs to quantify } \\
\text { de-identified data sets to } \\
\text { measure impact on } \\
\text { first-fill medication } \\
\text { adherence. }\end{array}$ & $\begin{array}{l}\text { - Evaluation of data from } \\
2008-2010 \\
\text { - Physicians who adopted } \\
\text { e-prescribing has } 10 \% \text { increase in } \\
\text { first-fill medication adherence } \\
\text { - Physicians who utilized } \\
\text { e-prescribing used the technology } \\
\text { route for up to } 40 \% \text { of their } \\
\text { prescriptions } \\
\text { - E-prescribing directly to the } \\
\text { pharmacy showed a } 11.7 \% \\
\text { increase in the number of } \\
\text { prescriptions that make it to the } \\
\text { pharmacy } \\
\text { - Increase in adherence will save } \\
\text { medical costs downstream in the } \\
\text { amounts of } \$ 3-\$ 10 \text { for a potential } \\
\text { total of } \$ 140 \text { billion dollars in } \\
\text { savings over the next } 10 \text { years. }\end{array}$ & $\begin{array}{c}\text { Health Management } \\
\text { Technology }\end{array}$ & B \\
\hline 2012 & $\begin{array}{c}\text { Westbrook, } \\
\text { Reckmann, Li, } \\
\text { Runciman, Burke, Lo, } \\
\text { Baysari, Braithwaite, } \\
\text { Day }\end{array}$ & $\begin{array}{l}\text { Effects of two commercial } \\
\text { electronic prescribing systems } \\
\text { on prescribing error rates in } \\
\text { hospital in-patients: a before } \\
\text { and after study }\end{array}$ & $\begin{array}{l}\text { Before and } \\
\text { after study }\end{array}$ & $\begin{array}{l}\text { Medication chart audit of } \\
\text { 3,291 admissions }\end{array}$ & $\begin{array}{c}\text {-Australian study at two } \\
\text { hospitals } \\
\text { - Controls were wards in same } \\
\text { two hospitals that did not use } \\
\text { electronic prescribing } \\
\text {-Procedural and clinical errors } \\
\text { were identified and the severity } \\
\text { measured } \\
\text { - Reductions in prescribing errors } \\
\text { seen } \\
\text {-Clinical error reductions were } \\
\text { limited due to lack of substantial } \\
\text { decision support but decline in } \\
\text { serious errors }\end{array}$ & PLOS Medicine & B \\
\hline 2013 & $\begin{array}{l}\text { Radley, Wasserman, } \\
\text { Olsho, Shoemaker, } \\
\text { Spranca, Bradshaw }\end{array}$ & $\begin{array}{l}\text { Reduction in medication } \\
\text { errors in hospitals due to } \\
\text { adoption of computerized } \\
\text { provider entry systems }\end{array}$ & $\begin{array}{l}\text { Systematic } \\
\text { literature } \\
\text { review and } \\
\text { meta-analysis }\end{array}$ & None & $\begin{array}{l}\text { - CPOE decreases likelihood of } \\
\text { an error on an order by } 48 \% \\
\text { - } 12.5 \% \text { reduction in medical } \\
\text { errors ( } \sim 17.4 \text { million/year) } \\
\text { - Only analyzed for acute-care } \\
\text { inpatient setting } \\
\text { - Unclear if this translates to } \\
\text { reduced harm to patients } \\
\text { - Data from 2006-2008 from } \\
4,701 \text { hospitals }\end{array}$ & $\begin{array}{c}\text { Journal of American } \\
\text { Medical Informatics } \\
\text { Association }\end{array}$ & B \\
\hline
\end{tabular}


Table 3. Summary of Articles:Clinician Adoption

\begin{tabular}{|c|c|c|c|c|c|c|c|}
\hline Year & Author & Title & Design & Intervention & Findings & Source & $\begin{array}{l}\text { Study } \\
\text { Grade }\end{array}$ \\
\hline 2003 & $\begin{array}{l}\text { Bartlett, Tamblyn, } \\
\text { Huang, Kawasumi, } \\
\text { Petrella, Dufour }\end{array}$ & $\begin{array}{l}\text { Evaluation of Standardized } \\
\text { Tasks for Primary Care } \\
\text { Physicians Using the } \\
\text { MOXXI Electronic } \\
\text { Prescribing and Integrated } \\
\text { Drug Management System }\end{array}$ & $\begin{array}{l}\text { Prospective } \\
\text { t-test }\end{array}$ & $\begin{array}{l}\text { Administering and } \\
\text { timing the completion of } \\
\text { a series of tasks by a } \\
\text { physician after a } 2 \text { week } \\
\text { training program }\end{array}$ & $\begin{array}{l}\text { - Physicians were given a } \\
\text { training session with the } \\
\text { e-prescribing software using } \\
\text { mock patients. } \\
\text { - They were then evaluated on } \\
\text { how quickly and efficiently they } \\
\text { were able to redo those tasks on } \\
\text { their own. } \\
\text { - Physicians were able to quickly } \\
\text { complete all tasks but only had } \\
\text { difficulty with changing the status } \\
\text { of a problem or with renewing } \\
\text { multiple prescriptions at once }\end{array}$ & $\begin{array}{c}\text { AMIA Annual } \\
\text { Symposium Archive }\end{array}$ & A \\
\hline 2005 & $\begin{array}{l}\text { Galt, Siracuse, Rule, } \\
\text { Clark, Taylor }\end{array}$ & $\begin{array}{l}\text { Physician Use of Hand-held } \\
\text { Computers for Drug } \\
\text { Information and Prescribing }\end{array}$ & $\begin{array}{l}\text { Randomized, } \\
\text { controlled } \\
\text { trial }\end{array}$ & $\begin{array}{l}\text { Primary care physicians } \\
\text { were given a PDA to } \\
\text { prescribe and the error } \\
\text { rates were assessed }\end{array}$ & $\begin{array}{l}\text {-Use for hand-held devices show } \\
\text { some validity } \\
\text { - Physicians feel that they can } \\
\text { see more patients } \\
\text { - Important to have positive } \\
\text { learning-related attitude } \\
\text {-Proper training needed }\end{array}$ & $\begin{array}{l}\text { Advances in Patient } \\
\text { Safety: From Research } \\
\text { to Implementation } \\
\text { (Book) }\end{array}$ & B \\
\hline 2005 & $\begin{array}{c}\text { Pizzi, Suh, Barone and } \\
\text { Nash }\end{array}$ & $\begin{array}{c}\text { Factors related to physicians' } \\
\text { adoption of electronic } \\
\text { prescribing: results from a } \\
\text { national survey }\end{array}$ & Quantitative study & Electronic survey & $\begin{array}{l}\text { - 1,104 responses recorded } \\
\text { - } 19 \% \text { of responses from } \\
\text { physicians who used } \\
\text { e-prescribing } \\
\text { - Adoption can be stimulated by } \\
\text { improvements in the technology } \\
\text { and an organizational } \\
\text { commitment }\end{array}$ & $\begin{array}{c}\text { American Journal of } \\
\text { Medical Quality }\end{array}$ & A \\
\hline 2007 & Gerstle, Lehmann & $\begin{array}{c}\text { Electronic prescribing } \\
\text { systems in pediatrics: the } \\
\text { rationale and functionality } \\
\text { requirements }\end{array}$ & $\begin{array}{c}\text { Technical } \\
\text { report }\end{array}$ & $\begin{array}{l}\text { Report discusses use of } \\
\text { e-prescribing in } \\
\text { pediatrics }\end{array}$ & $\begin{array}{l}\text { - Evaluates e-prescribing in all } \\
\text { aspects (from costs to technical } \\
\text { issues) }\end{array}$ & Pediatrics & B \\
\hline 2007 & $\begin{array}{l}\text { Grossman, Gerland, } \\
\text { Reed and Fahlman }\end{array}$ & $\begin{array}{l}\text { Physician's experiences } \\
\text { using commercial } \\
\text { e-prescribing systems }\end{array}$ & Discussion & $\begin{array}{l}44 \text { discussions from } \\
\text { respondents in } \\
\text { two-person teams in } 26 \\
\text { organizations via } \\
\text { telephone }\end{array}$ & $\begin{array}{c}\text { - 2/3 of practices used EMR } \\
\text { (integrated system) } \\
\text { - 1/3 stand-alone system } \\
\text { - Issue with maintaining patient } \\
\text { medication lists } \\
\text { - Lack of decision support } \\
\text { - Difficulty obtaining accurate } \\
\text { patient-specific formulary } \\
\text { information } \\
\text { - Limited connectivity with } \\
\text { pharmacies and mail-order PBMs }\end{array}$ & Health Affairs & B \\
\hline
\end{tabular}




\begin{tabular}{|c|c|c|c|c|c|c|c|}
\hline & & & & & $\begin{array}{l}\text { - Not enough staff to maintain } \\
\text { and support IT after } \\
\text { implementation } \\
\text { - All respondents believe } \\
\text { e-prescribing improves safety and } \\
\text { quality while increasing practice } \\
\text { efficiency } \\
\end{array}$ & & \\
\hline 2008 & $\begin{array}{l}\text { Fischer, Vogeli, } \\
\text { Stedman, Ferris, } \\
\text { Brookhart, Weissman }\end{array}$ & $\begin{array}{l}\text { Effect of electronic } \\
\text { prescribing with formulary } \\
\text { decision support on } \\
\text { medication use and cost }\end{array}$ & $\begin{array}{c}\text { Pre-post } \\
\text { study with } \\
\text { concurrent controls }\end{array}$ & $\begin{array}{c}\text { Analysis of } \\
\text { administrative data in } 2 \\
\text { large Massachusetts } \\
\text { insurers }\end{array}$ & $\begin{array}{l}\text { - Reduced spending on } \\
\text { medications due to formulary } \\
\text { decision support } \\
\text { - Physicians more likely to } \\
\text { prescribe tier } 1 \text { drugs } \\
\text { - } \$ 845,000 / 100,000 \text { patients } \\
\text { saving } \\
\end{array}$ & $\begin{array}{l}\text { Archives of Internal } \\
\text { Medicine }\end{array}$ & A \\
\hline 2008 & Rupp, Warholak & $\begin{array}{l}\text { Evaluation of e-prescribing } \\
\text { in chain community } \\
\text { pharmacy practice } \\
\text { recommendations }\end{array}$ & $\begin{array}{c}\text { Descriptive, } \\
\text { nonexperimental, } \\
\text { cross-sectional study }\end{array}$ & $\begin{array}{c}\text { Self-administered survey } \\
\text { and interviews of key } \\
\text { pharmacy operations and } \\
\text { information technology } \\
\text { management }\end{array}$ & $\begin{array}{c}\bullet 1,094 \text { surveys returned. } \\
\bullet \text { E-prescriptions were preferred } \\
\text { in each of the } 7 \text { desired outcomes } \\
\text { of care. } \\
\bullet \text { Benefits: legibility, improved } \\
\text { speed on efficiency of processing } \\
\text { Disadvantage: prescribing errors } \\
\text { due to lack of training }\end{array}$ & $\begin{array}{c}\text { Journal of the } \\
\text { American Pharmacists } \\
\text { Association }\end{array}$ & B \\
\hline 2010 & $\begin{array}{l}\text { Desroches, Agarwal, } \\
\text { Angst, and Fischer }\end{array}$ & $\begin{array}{l}\text { Differences between } \\
\text { integrated and stand-alone } \\
\text { E-prescribing systems have } \\
\text { implications for future use }\end{array}$ & Qualitative analysis & $\begin{array}{l}\text { Surveyed physicians } \\
\text { about use of } \\
\text { e-prescribing in an } \\
\text { outpatient setting }\end{array}$ & $\begin{array}{l}\bullet \text { Physicians with access to } \\
\text { patient's drug history were more } \\
\text { likely to review it before } \\
\text { prescribing a new medication. } \\
\text { - This percentage was higher in } \\
\text { those using integrated systems. }\end{array}$ & $\begin{array}{l}\text { Health Affairs } \\
\text { (Milwood) }\end{array}$ & B \\
\hline 2010 & $\begin{array}{l}\text { Tseng, Brook, } \\
\text { Alexander, Hixon, } \\
\text { Dudley, et al. }\end{array}$ & $\begin{array}{c}\text { Health Information } \\
\text { Technology and Physician's } \\
\text { Knowledge of Drug Costs }\end{array}$ & $\begin{array}{c}\text { Descriptive, } \\
\text { nonexperimental, } \\
\text { cross-sectional study }\end{array}$ & $\begin{array}{c}\text { Statewide survey of } 247 \\
\text { primary care physicians } \\
\text { regarding IT use and } \\
\text { self-reported knowledge } \\
\text { of formularies, } \\
\text { copayments, and retail } \\
\text { prices } \\
\end{array}$ & $\begin{array}{l}-80 \% \text { of physicians used IT but } \\
\text { less than } 20 \% \text { knew drug costs. } \\
\text { - Less than } 10 \% \text { knew about } \\
\text { formularies. } \\
\text { - Recommendation: IT design to } \\
\text { consider easily accessible cost } \\
\text { information at point of care } \\
\end{array}$ & $\begin{array}{c}\text { American Journal of } \\
\text { Managed Care }\end{array}$ & B \\
\hline 2011 & $\begin{array}{l}\text { Frolich, Zorina, } \\
\text { Fontana, } \\
\text { Kullak-Ublick, } \\
\text { Vollenweider, } \\
\text { Russmann }\end{array}$ & $\begin{array}{l}\text { Evaluation of medication } \\
\text { safety in the discharge } \\
\text { medication of } 509 \text { surgical } \\
\text { inpatients using electronic } \\
\text { prescription support software } \\
\text { and an extended operational } \\
\text { interaction classification }\end{array}$ & $\begin{array}{c}\text { Cross-sectional } \\
\text { study }\end{array}$ & $\begin{array}{c}\text { Retrospective } \\
\text { identification of drug } \\
\text { interactions in patients' } \\
\text { discharge medication }\end{array}$ & $\begin{array}{c}\bullet 2,558 \text { of } 2,729 \text { prescriptions } \\
\text { generated interaction alerts and } \\
1,849 \text { comments } \\
\bullet 10 \text { high danger } \\
\text { (contraindications) based on } \\
\text { ORCA criteria } \\
\text { - } 551 \text { average danger alerts based } \\
\text { on ORCA criteria ( } 77 \\
\text { provisionally contraindicated } \\
\text { combos, } 310 \text { conditional and } 164 \\
\text { minimal risk of adverse } \\
\end{array}$ & $\begin{array}{l}\text { European Journal of } \\
\text { Clinical Pharmacology }\end{array}$ & B \\
\hline
\end{tabular}




\begin{tabular}{|c|c|c|c|c|c|c|c|}
\hline & & & & & $\begin{array}{l}\text { outcomes) } \\
\text {-56 did not have recommended } \\
\text { dose adjustment for impaired } \\
\text { renal function } \\
\text { - Need to reduce over-alerting } \\
\text { and improve usability to be more } \\
\text { efficacious in preventing adverse } \\
\text { drug events }\end{array}$ & & \\
\hline 2011 & $\begin{array}{l}\text { Gaylin, Moiduddin, } \\
\text { Mohamoud, Lundeen, } \\
\text { Kelly }\end{array}$ & $\begin{array}{l}\text { Public attitudes about health } \\
\text { information technology, and } \\
\text { its relationship to health care } \\
\text { quality, costs and privacy }\end{array}$ & $\begin{array}{l}\text { Mulitvariate logistic } \\
\text { regression analysis }\end{array}$ & $\begin{array}{l}\text { Random-digit-dial } \\
\text { sample telephone survey } \\
\text { and interview }\end{array}$ & $\begin{array}{l}\text { - } 78 \% \text { of responses favored used } \\
\text { of electronic medical records and } \\
\text { could improve care and reduce } \\
\text { costs } \\
\text { - Benefits outweigh privacy risks }\end{array}$ & $\begin{array}{l}\text { Health Service } \\
\text { Research }\end{array}$ & A \\
\hline 2011 & $\begin{array}{l}\text { Grossman, Cross, } \\
\text { Boukus, Cohen }\end{array}$ & $\begin{array}{l}\text { Transmitting and processing } \\
\text { electronic prescriptions: } \\
\text { experiences of physician } \\
\text { practices and pharmacies }\end{array}$ & Qualitative analysis & $\begin{array}{c}\text { Telephone interviews } \\
\text { with physician practices, } \\
\text { community pharmacies } \\
\text { and mail order } \\
\text { pharmacies using } \\
\text { Surescripts }\end{array}$ & $\begin{array}{l}\text {-Most parties were satisfied with } \\
\text { the use of electronic transmission } \\
\text { but there were system limitations } \\
\text { that prevented electronic } \\
\text { transmission and required a fax or } \\
\text { phoned in prescription. } \\
\text { - Inconsistent renewal processes } \\
\text { by physicians required pharmacy } \\
\text { staff to complete or edit fields }\end{array}$ & $\begin{array}{l}\text { Journal of American } \\
\text { Medical Informatics } \\
\text { Association }\end{array}$ & $\mathrm{B}$ \\
\hline 2011 & Lapane, Rosen, Dube & $\begin{array}{l}\text { Perceptions of e-prescribing } \\
\text { efficiencies and inefficiencies } \\
\text { in ambulatory care }\end{array}$ & $\begin{array}{l}\text { Mixed method study } \\
\text { design using } \\
\text { qualitative data }\end{array}$ & $\begin{array}{c}\text { Focus groups reported } \\
\text { back via survey on one } \\
\text { of six software packages. } \\
\text { Results were analyzed } \\
\text { and coded using NVivo } \\
\text { software } \\
\end{array}$ & $\begin{array}{l}\bullet 64 \% \text { physicians viewed } \\
\text { e-prescribing as very efficient. } \\
\bullet \text { Next preferred at } 25 \% \text { was } \\
\text { computer generated fax. }\end{array}$ & $\begin{array}{l}\text { International Journal of } \\
\text { Medical Informatics }\end{array}$ & B \\
\hline 2011 & $\begin{array}{l}\text { Spina, Glassman, } \\
\text { Simon, Lanto, Lee, } \\
\text { Cunningham, Good }\end{array}$ & $\begin{array}{l}\text { Potential safety gaps in order } \\
\text { entry and automated drug } \\
\text { alerts: a nationwide survey of } \\
\text { VA physician self-reported } \\
\text { practices with computerized } \\
\text { order entry }\end{array}$ & $\begin{array}{c}\text { Cross-sectional } \\
\text { study }\end{array}$ & $\begin{array}{l}\text { National survey of VA } \\
\text { physicians containing } 35 \\
\text { questions in } 4 \text { domains }\end{array}$ & $\begin{array}{l}\text { - } 90 \% \text { felt improved prescribing } \\
\text { safety } \\
\text { - } 48 \% \text { felt critical drug-drug } \\
\text { interactions as useful } \\
\text {-Medical specialists found less } \\
\text { useful } \\
\end{array}$ & Medical Care & B \\
\hline 2012 & $\begin{array}{l}\text { Crosson, Schueth, } \\
\text { Issacson and Bell }\end{array}$ & $\begin{array}{l}\text { Early adopters of electronic } \\
\text { prescribing struggle to make } \\
\text { meaningful use of formulary } \\
\text { checks and medication } \\
\text { history documentation }\end{array}$ & $\begin{array}{l}\text { Multimethod } \\
\text { comparative } \\
\text { case study }\end{array}$ & $\begin{array}{l}\text { Observation of } \\
\text { prescription workflow } \\
\text { during implementation } \\
\text { of stand-alone } \\
\text { e-prescribing program } \\
\text { and conduction of } \\
\text { interviews with } \\
\text { physicians and staff }\end{array}$ & $\begin{array}{l}\text { - Incomplete or challenges with } \\
\text { formulary checks or patient } \\
\text { history documentation led to } \\
\text { prescriber distrust } \\
\text { - Further leads to unwillingness } \\
\text { to rely on e-prescribing-based } \\
\text { information and prefers } \\
\text { dependence on paper charts } \\
\text { - Greater data accuracy and } \\
\text { completeness required to improve } \\
\text { safety and efficacy } \\
\end{array}$ & $\begin{array}{l}\text { Journal of the } \\
\text { American Board of } \\
\text { Family Medicine }\end{array}$ & $\mathrm{B}$ \\
\hline 2012 & Odukoya, Chui & Relationship between & Cross-sectional & Thinking aloud protocols & -Two factors influencing & Journal of the & B \\
\hline
\end{tabular}




\begin{tabular}{|c|c|c|c|c|c|c|c|}
\hline & & $\begin{array}{c}\text { e-prescriptions and } \\
\text { community pharmacy } \\
\text { workflow }\end{array}$ & $\begin{array}{l}\text { qualitative } \\
\text { study }\end{array}$ & $\begin{array}{l}\text { and pharmacy group } \\
\text { interviews of } 16 \\
\text { pharmacists and } 14 \\
\text { pharmacy technicians }\end{array}$ & $\begin{array}{l}\text { workflow: software issues and } \\
\text { informal training }\end{array}$ & $\begin{array}{l}\text { American Pharmacists } \\
\text { Association }\end{array}$ & \\
\hline 2012 & $\begin{array}{l}\text { Thomas, Kim, } \\
\text { McDonald, Kreiner, } \\
\text { Kelleher, Blackman, } \\
\text { Kaufman, Carrow }\end{array}$ & $\begin{array}{c}\text { Prescribers' expectations and } \\
\text { barriers to electronic } \\
\text { prescribing of controlled } \\
\text { substances }\end{array}$ & Survey & $\begin{array}{l}\text { On-site interviews with } \\
\text { physicians and providers } \\
\text { regarding e-prescribing } \\
\text { controlled substances. }\end{array}$ & $\begin{array}{l}\text { - } 246 \text { practitioners participated } \\
\text { with } 63 \% \text { male and } 90 \% \text { white. } \\
\text { - Most issues were due to errors, } \\
\text { pharmacy call-backs, diversion. } \\
\text { - Physicians found that } \\
\text { expectation to carrying a security } \\
\text { authentication token at all times } \\
\text { would be burdensome and deter } \\
\text { from adopting e-prescribing. }\end{array}$ & $\begin{array}{c}\text { Journal of American } \\
\text { Medical Informatics } \\
\text { Association }\end{array}$ & A \\
\hline 2012 & $\begin{array}{l}\text { Weingart, Mattsson, } \\
\text { Zhu, Shulman, Hassett }\end{array}$ & $\begin{array}{c}\text { Improving Electronic Oral } \\
\text { Chemotherapy Prescription: } \\
\text { Can We Build a Safer } \\
\text { System? }\end{array}$ & Prospective study & $\begin{array}{l}\text { Review of generated } \\
\text { electronic prescriptions } \\
\text { for surveyed ease of use }\end{array}$ & $\begin{array}{l}\text { - } 6,673 \text { prescriptions generated } \\
\text { using weight and BSA-based } \\
\text { dosing. } \\
\text { - 5\% of prescriptions generated } \\
\text { dose-limit warnings but were } \\
\text { disregarded by physicians } \\
\text { because current dosing } \\
\text { recommendations exceeded } \\
\text { dose-limit warnings. } \\
\text { - Clinicians still needed to } \\
\text { customize their directions using } \\
\text { the free text field for } 64 \% \text { of the } \\
\text { prescriptions. }\end{array}$ & $\begin{array}{c}\text { Journal of Oncology } \\
\text { Practice }\end{array}$ & A \\
\hline 2013 & $\begin{array}{c}\text { Ancker, Silver, Miller, } \\
\text { Kaushal }\end{array}$ & $\begin{array}{l}\text { Consumer experience with } \\
\text { and attitudes towards health } \\
\text { information technology: a } \\
\text { nationwide survey }\end{array}$ & $\begin{array}{l}\text { Mulitvariate logistic } \\
\text { regression analysis }\end{array}$ & $\begin{array}{c}\text { Random digit-dial } \\
\text { survey of } 1,603 \text { eligible } \\
\text { participants }\end{array}$ & $\begin{array}{c}\bullet n=1,000 \text { participation } \\
\bullet 66 \% \text { positive view on EHR and } \\
\text { its likelihood to improve quality } \\
\text { of care; } 79 \% \text { positive view on } \\
\text { health information exchange } \\
\bullet \text { Not associated with privacy } \\
\text { concerns } \\
\end{array}$ & $\begin{array}{l}\text { Journal of the } \\
\text { American Medical } \\
\text { Informatics } \\
\text { Association }\end{array}$ & A \\
\hline 2013 & $\begin{array}{l}\text { Jariwala, Holmes, } \\
\text { Banahan, McCaffrey }\end{array}$ & $\begin{array}{l}\text { Factors that physicians find } \\
\text { encouraging and } \\
\text { discouraging about electronic } \\
\text { prescribing: a quantitative } \\
\text { study }\end{array}$ & Quantitative study & $\begin{array}{c}\text { Cross-sectional study } \\
\text { using an internet-based } \\
\text { survey to a national } \\
\text { sample of primary care } \\
\text { physicians }\end{array}$ & $\begin{array}{c}\text { - } 443 \text { surveys received } \\
\text { - Pre-implementation and cost } \\
\text { factors found to be most } \\
\text { discouraging } \\
\text { - Software features most } \\
\text { encouraging } \\
\text { - Facilitation of adoption } \\
\text { depends on targeting factors that } \\
\text { are salient to physicians } \\
\end{array}$ & $\begin{array}{c}\text { Journal of American } \\
\text { Medical Informatics } \\
\text { Association }\end{array}$ & A \\
\hline 2013 & Johnson, Lehmann & $\begin{array}{c}\text { Electronic prescribing in } \\
\text { pediatrics: toward safer and } \\
\text { more effective medication } \\
\text { management }\end{array}$ & $\begin{array}{l}\text { Technical } \\
\text { report }\end{array}$ & Review of pediatric data & $\begin{array}{l}\text { - Limited data for role of } \\
\text { e-prescribing } \\
\text { - Existing data shows positive } \\
\text { response for mitigating med }\end{array}$ & Pediatrics & $\mathrm{C}$ \\
\hline
\end{tabular}




\begin{tabular}{|c|c|c|c|c|c|c|c|}
\hline & & & & & $\begin{array}{l}\text { errors, improve communication } \\
\text { with pharmacy and better } \\
\text { medication adherence } \\
\text { - American Academy of } \\
\text { Pediatrics recommends the } \\
\text { adoption of e-prescribing systems } \\
\text { with pediatric functionality }\end{array}$ & & \\
\hline 2013 & $\begin{array}{l}\text { Thomas, Kim, } \\
\text { Kelleher, Nikitin, } \\
\text { Kreiner, McDonald, } \\
\text { Carrow }\end{array}$ & $\begin{array}{l}\text { Early experience with } \\
\text { electronic prescribing of } \\
\text { controlled substances in a } \\
\text { community setting }\end{array}$ & Prospective analysis & $\begin{array}{l}\text { Survey given to } \\
\text { physicians before and } \\
\text { after implementation of } \\
\text { electronic prescribing of } \\
\text { controlled substances } \\
\text { (EPCS) }\end{array}$ & $\begin{array}{l}\text { - 70/102 prescribers sent at least } \\
\text { one controlled substance } \\
\text { - Found to be less burdensome } \\
\text { than previously thought } \\
\text { - Barrier is limited pharmacy } \\
\text { participation and unreliable } \\
\text { technology instances }\end{array}$ & $\begin{array}{c}\text { Journal of American } \\
\text { Medical Informatics } \\
\text { Association }\end{array}$ & B \\
\hline
\end{tabular}

Table 4. Summary of Articles:Limitations

\begin{tabular}{|c|c|c|c|c|c|c|c|}
\hline Year & Author & Title & Design & Intervention & Findings & Source & $\begin{array}{l}\text { Study } \\
\text { Grade }\end{array}$ \\
\hline 2005 & $\begin{array}{l}\text { Galt, Siracuse, Rule, } \\
\text { Clark, Taylor }\end{array}$ & $\begin{array}{l}\text { Physician Use of Hand-held } \\
\text { Computers for Drug } \\
\text { Information and Prescribing }\end{array}$ & $\begin{array}{l}\text { Randomized, } \\
\text { controlled } \\
\text { trial }\end{array}$ & $\begin{array}{l}\text { Primary care physicians } \\
\text { were given a PDA to } \\
\text { prescribe and the error } \\
\text { rates were assessed }\end{array}$ & $\begin{array}{l}\text {-Use for hand-held devices show } \\
\text { some validity } \\
\text { - Physicians feel that they can } \\
\text { see more patients } \\
\text { - Important to have positive } \\
\text { learning-related attitude } \\
\text {-Proper training needed }\end{array}$ & $\begin{array}{l}\text { Advances in Patient } \\
\text { Safety: From Research } \\
\text { to Implementation } \\
\text { (Book) }\end{array}$ & B \\
\hline 2005 & McMullin & $\begin{array}{c}\text { Standardization is Necessary } \\
\text { in the Methods to Assess the } \\
\text { Value of Electronic } \\
\text { Prescribing Systems }\end{array}$ & Post-hoc analysis & $\begin{array}{l}\text { Follow-up study on drug } \\
\text { utilization and costs } \\
\text { based on script volume } \\
\text { as reported by use of an } \\
\text { electronic prescribing } \\
\text { system }\end{array}$ & $\begin{array}{l}\text { - While the study mainly focuses } \\
\text { on the use of one particular } \\
\text { e-prescribing program, it } \\
\text { mentions the need for } \\
\text { standardization to better } \\
\text { accurately assess the value of } \\
\text { certain programs over another. }\end{array}$ & JMCP & B \\
\hline 2006 & Grimsmo & $\begin{array}{c}\text { Electronic prescriptions - } \\
\text { without side-effects? }\end{array}$ & $\begin{array}{c}\text { International } \\
\text { literature analysis }\end{array}$ & Literature review & $\begin{array}{l}\text { - Need to continually prepare for } \\
\text { unknown risks with electronic } \\
\text { prescribing } \\
\text { - Need for constant system } \\
\text { testing and evaluation after } \\
\text { implementation } \\
\text { - New system may change or } \\
\text { unintentionally put aside } \\
\text { well-established safety routines }\end{array}$ & Norwegian Article & B \\
\hline 2006 & $\begin{array}{c}\text { Halamka, Ascenzo, } \\
\text { Bates, Berry, Debor, et } \\
\text { al. }\end{array}$ & $\begin{array}{l}\text { E-Prescribing collaboration } \\
\text { in Massachusetts: early } \\
\text { experiences from regional } \\
\text { prescribing projects }\end{array}$ & Report & None & $\begin{array}{c}\text { - Evaluation of barriers to } \\
\text { e-prescribing } \\
\text { - Previous negative technology } \\
\text { experiences, costs, lost } \\
\text { productivity, competing }\end{array}$ & $\begin{array}{l}\text { Journal of the } \\
\text { American Medical } \\
\text { Informatics } \\
\text { Association }\end{array}$ & $\mathrm{C}$ \\
\hline
\end{tabular}




\begin{tabular}{|c|c|c|c|c|c|c|c|}
\hline & & & & & $\begin{array}{l}\text { priorities, change management } \\
\text { issues, interoperability } \\
\text { limitations, IT requirements, } \\
\text { standards limitations, waiting for } \\
\text { an "all-in-one solution" and } \\
\text { confusion about competing } \\
\text { product offerings }\end{array}$ & & \\
\hline 2007 & $\begin{array}{l}\text { Donyai, O'Grady, } \\
\text { Jacklin, Barber, } \\
\text { Franklin }\end{array}$ & $\begin{array}{l}\text { The effects of electronic } \\
\text { prescribing on the quality of } \\
\text { prescribing }\end{array}$ & $\begin{array}{c}\text { Prospective } \\
\text { intervention analysis }\end{array}$ & $\begin{array}{l}\text { Recording of pharmacy } \\
\text { interventions during a } 4 \\
\text { week period before and } \\
\text { after implementation of } \\
\text { electronic prescribing }\end{array}$ & $\begin{array}{c}\bullet \text { UK study } \\
\bullet \text { E-prescribing reduced } \\
\text { pharmacist intervention }(73 \rightarrow 45) \\
\text { as well as prescribing errors }(94 \\
\rightarrow 48) \\
\bullet \text { Pharmacy needs to develop } \\
\text { ways to complement use of } \\
\text { electronic prescribing rather than } \\
\text { duplicate the benefits of it }\end{array}$ & $\begin{array}{l}\text { British Journal of } \\
\text { Clinical Pharmacology }\end{array}$ & B \\
\hline 2007 & Gerstle, Lehmann & $\begin{array}{c}\text { Electronic prescribing } \\
\text { systems in pediatrics: the } \\
\text { rationale and functionality } \\
\text { requirements } \\
\end{array}$ & $\begin{array}{l}\text { Technical } \\
\text { report }\end{array}$ & $\begin{array}{l}\text { Report discusses use of } \\
\text { e-prescribing in } \\
\text { pediatrics }\end{array}$ & $\begin{array}{l}\text { - Evaluates e-prescribing in all } \\
\text { aspects (from costs to technical } \\
\text { issues) }\end{array}$ & Pediatrics & B \\
\hline 2007 & $\begin{array}{l}\text { Grossman, Gerland, } \\
\text { Reed and Fahlman }\end{array}$ & $\begin{array}{l}\text { Physician's experiences } \\
\text { using commercial } \\
\text { e-prescribing systems }\end{array}$ & Discussion & $\begin{array}{l}44 \text { discussions from } \\
\text { respondents in } \\
\text { two-person teams in } 26 \\
\text { organizations via } \\
\text { telephone }\end{array}$ & $\begin{array}{l}\text { - 2/3 of practices used EMR } \\
\text { (integrated system) } \\
\text { - 1/3 stand-alone system } \\
\text { - Issue with maintaining patient } \\
\text { medication lists } \\
\text { • Lack of decision support } \\
\text { - Difficulty obtaining accurate } \\
\text { patient-specific formulary } \\
\text { information } \\
\text { - Limited connectivity with } \\
\text { pharmacies and mail-order PBMs } \\
\text { - Not enough staff to maintain } \\
\text { and support IT after } \\
\text { implementation } \\
\text { - All respondents believe } \\
\text { e-prescribing improves safety and } \\
\text { quality while increasing practice } \\
\text { efficiency }\end{array}$ & Health Affairs & B \\
\hline 2007 & Scalise & The case for e-prescribing & Analysis & None & $\begin{array}{l}\text { - Lost productivity due to time } \\
\text { required to print PDA generated } \\
\text { prescriptions and fax pharmacies } \\
\text { (applies to controlled } \\
\text { medications) } \\
\text { - Program functionality } \\
\text { confusion } \\
\text { - Cost - software/hardware cost }\end{array}$ & $\begin{array}{l}\text { Hospital and Health } \\
\text { Networks }\end{array}$ & $\mathrm{B}$ \\
\hline
\end{tabular}




\begin{tabular}{|c|c|c|c|c|c|c|c|}
\hline & & & & & $\begin{array}{c}\text { ranges from } \$ 1,500 \text { - } \\
\$ 4,000 / \text { physician } \\
\text { - Advanced systems with } \\
\text { complex alerts and reminders are } \\
\$ 29,000 / \text { physician in the first year } \\
\text { and } \$ 4,000 \text { annually thereafter } \\
\text { - Waiting for an all-in-one } \\
\text { solution before investing in a } \\
\text { product }\end{array}$ & & \\
\hline 2007 & $\begin{array}{l}\text { Vanderbush, } \\
\text { Anderson, Fant, } \\
\text { Fujisaki, Malone, } \\
\text { Price, et al. }\end{array}$ & $\begin{array}{l}\text { Implementing Pharmacy } \\
\text { Informatics in College } \\
\text { Curricula: The AACP } \\
\text { Technology in Pharmacy in } \\
\text { Education and Learning } \\
\text { Special Interest Group }\end{array}$ & Discussion & None & $\begin{array}{l}\text { - Stressing the importance of } \\
\text { giving students more awareness } \\
\text { and use of informatics in the } \\
\text { practice of pharmacy while still in } \\
\text { school. }\end{array}$ & $\begin{array}{l}\text { American Journal of } \\
\text { Pharmaceutical } \\
\text { Education }\end{array}$ & $\mathrm{D}$ \\
\hline 2008 & Cusack & $\begin{array}{l}\text { Electronic health records and } \\
\text { electronic prescribing: } \\
\text { promise and pitfalls }\end{array}$ & Analysis & Survey & $\begin{array}{l}\text { - Evaluation of implementation } \\
\text { issues } \\
\text { - Push for investment due to } \\
\text { pay-per-performance incentives } \\
\text { • However, adopting } \\
\text { e-prescribing is a difficult task } \\
\text { due to costly investment and } \\
\text { systems disruption }\end{array}$ & $\begin{array}{l}\text { Obstetrics and } \\
\text { gynecology Clinics of } \\
\text { North America }\end{array}$ & B \\
\hline 2008 & $\begin{array}{c}\text { Devine, } \\
\text { Wilson-Norton, } \\
\text { Lawless, Hansen, } \\
\text { Hollingworth, Fisk, } \\
\text { Sullivan }\end{array}$ & $\begin{array}{l}\text { Implementing an Ambulatory } \\
\text { e-Prescribing System: } \\
\text { Strategies Employed and } \\
\text { Lessons Learned to Minimize } \\
\text { Unintended Consequences }\end{array}$ & Analysis & None & $\begin{array}{l}\text { CPOE improves quality of care } \\
\text { and reduces med errors } \\
\bullet \text { Need an integrated, } \\
\text { community-based system for } \\
\text { adoption } \\
\text { - Need for ongoing training } \\
\text { - Key is team-oriented culture } \\
\text { (bridge between all points of } \\
\text { healthcare) }\end{array}$ & $\begin{array}{l}\text { Advances in Patient } \\
\text { Safety: Alternative } \\
\text { Approaches (Volume } \\
\text { 4: Technology and } \\
\text { Medication Safety) }\end{array}$ & B \\
\hline 2008 & Rupp, Warholak & $\begin{array}{l}\text { Evaluation of e-prescribing } \\
\text { in chain community } \\
\text { pharmacy practice } \\
\text { recommendations }\end{array}$ & $\begin{array}{c}\text { Descriptive, } \\
\text { nonexperimental, } \\
\text { cross-sectional study }\end{array}$ & $\begin{array}{l}\text { Self-administered survey } \\
\text { and interviews of key } \\
\text { pharmacy operations and } \\
\text { information technology } \\
\text { management }\end{array}$ & $\begin{array}{c}\bullet 1,094 \text { surveys returned. } \\
\text { •E-prescriptions were preferred } \\
\text { in each of the } 7 \text { desired outcomes } \\
\text { of care. } \\
\bullet \text { Benefits: legibility, improved } \\
\text { speed on efficiency of processing } \\
\text { Disadvantage: prescribing errors } \\
\text { due to lack of training }\end{array}$ & $\begin{array}{c}\text { Journal of the } \\
\text { American Pharmacists } \\
\text { Association }\end{array}$ & B \\
\hline 2009 & $\begin{array}{c}\text { Balfour, Evans, } \\
\text { Januska, Lee, Lewis, } \\
\text { Nolan, Noga, Stemple, } \\
\text { Thapar }\end{array}$ & $\begin{array}{c}\text { Health Information } \\
\text { Technology - Results from a } \\
\text { Roundtable Discussion }\end{array}$ & Discussion & None & $\begin{array}{l}\text { - Challenges to adoption of } \\
\text { e-prescribing are high costs, } \\
\text { resistance to change, misaligned } \\
\text { incentives and fractured payment } \\
\text { system } \\
\text { - Benefits: avoid costs from }\end{array}$ & $\mathrm{JMCP}$ & $\mathrm{C}$ \\
\hline
\end{tabular}




\begin{tabular}{|c|c|c|c|c|c|c|c|}
\hline & & & & & $\begin{array}{l}\text { adverse drug events } \\
\text { - Impact on cost exceed a saving } \\
\text { of } \$ 800 \text { million over a } 5 \text { year } \\
\text { period by prescribers, dispenses } \\
\text { and beneficiaries } \\
\text { - E-rx transactions have risen } 3 x \\
\text { from } 2007 \text { to } 2008 \text { with over } 100 \\
\text { million transactions (started with } \\
700,000 \text { in } 2004)\end{array}$ & & \\
\hline 2009 & Warholak, Rupp & $\begin{array}{c}\text { Analysis of community chain } \\
\text { pharmacists' on electronic } \\
\text { prescriptions }\end{array}$ & $\begin{array}{c}\text { Descriptive, } \\
\text { nonexperimental, } \\
\text { cross-sectional study }\end{array}$ & $\begin{array}{l}\text { Panel of pharmacists } \\
\text { reported medication } \\
\text { therapy interventions } \\
\text { using a standardized } \\
\text { protocol }\end{array}$ & $\begin{array}{c}\bullet \text { Reviewed reasons why } \\
\text { interventions were made based on } \\
\text { whether they were new or refill } \\
\text { prescriptions. } \\
\bullet \text { Most common reason: omitted } \\
\text { information from physician } \\
\text { (especially directions) or dosing } \\
\text { errors } \\
\end{array}$ & $\begin{array}{c}\text { Journal of the } \\
\text { American Pharmacists } \\
\text { Association }\end{array}$ & B \\
\hline 2010 & $\begin{array}{l}\text { Savage, Cornford, } \\
\text { Klecun, Barber, } \\
\text { Clifford, Franklin }\end{array}$ & $\begin{array}{c}\text { Medication errors with } \\
\text { electronic prescribing }(\mathrm{eP}) \text { : } \\
\text { Two views of the same } \\
\text { picture }\end{array}$ & $\begin{array}{l}\text { Structured, } \\
\text { quantitative } \\
\text { retrospective study } \\
\text { AND } \\
\text { qualitative } \\
\text { retrospective } \\
\text { method }\end{array}$ & $\begin{array}{l}\text { Interviews with staff and } \\
\text { patients on medication } \\
\text { incidents in London } \\
\text { hospitals two years after } \\
\text { e-prescribing } \\
\text { implementation OR } \\
\text { retrospective analysis of } \\
\text { data through electronic } \\
\text { system }\end{array}$ & $\begin{array}{l}\text { - Qualitative studies provided } \\
\text { more understanding of processes } \\
\text { and insight as to why medication } \\
\text { errors happen } \\
\text { - Electronic prescribing errors } \\
\text { resulted from improper med } \\
\text { reconciliation at admission and } \\
\text { department transfer } \\
\text { - Lack of allergy warnings to get } \\
\text { drug interactions } \\
\text { - Non-user friendly order entry } \\
\text { screen and lack of decision } \\
\text { support }\end{array}$ & $\begin{array}{l}\text { BMC Health Services } \\
\text { Research }\end{array}$ & B \\
\hline 2011 & $\begin{array}{l}\text { Frolich, Zorina, } \\
\text { Fontana, } \\
\text { Kullak-Ublick, } \\
\text { Vollenweider, } \\
\text { Russmann }\end{array}$ & $\begin{array}{l}\text { Evaluation of medication } \\
\text { safety in the discharge } \\
\text { medication of } 509 \text { surgical } \\
\text { inpatients using electronic } \\
\text { prescription support software } \\
\text { and an extended operational } \\
\text { interaction classification }\end{array}$ & $\begin{array}{c}\text { Cross-sectional } \\
\text { study }\end{array}$ & $\begin{array}{c}\text { Retrospective } \\
\text { identification of drug } \\
\text { interactions in patients' } \\
\text { discharge medication }\end{array}$ & $\begin{array}{c}\bullet 2,558 \text { of } 2,729 \text { prescriptions } \\
\text { generated interaction alerts and } \\
1,849 \text { comments } \\
\bullet 10 \text { high danger } \\
\text { (contraindications) based on } \\
\text { ORCA criteria } \\
\text { - } 551 \text { average danger alerts based } \\
\text { on ORCA criteria (77 } \\
\text { provisionally contraindicated } \\
\text { combos, } 310 \text { conditional and } 164 \\
\text { minimal risk of adverse } \\
\text { outcomes) } \\
\text {-56 did not have recommended } \\
\text { dose adjustment for impaired } \\
\text { renal function } \\
\text { - Need to reduce over-alerting }\end{array}$ & $\begin{array}{l}\text { European Journal of } \\
\text { Clinical Pharmacology }\end{array}$ & B \\
\hline
\end{tabular}




\begin{tabular}{|c|c|c|c|c|c|c|c|}
\hline & & & & & $\begin{array}{l}\text { and improve usability to be more } \\
\text { efficacious in preventing adverse } \\
\text { drug events }\end{array}$ & & \\
\hline 2011 & $\begin{array}{l}\text { Grossman, Cross, } \\
\text { Boukus, Cohen }\end{array}$ & $\begin{array}{l}\text { Transmitting and processing } \\
\text { electronic prescriptions: } \\
\text { experiences of physician } \\
\text { practices and pharmacies }\end{array}$ & Qualitative analysis & $\begin{array}{c}\text { Telephone interviews } \\
\text { with physician practices, } \\
\text { community pharmacies } \\
\text { and mail order } \\
\text { pharmacies using } \\
\text { Surescripts }\end{array}$ & $\begin{array}{l}\text {-Most parties were satisfied with } \\
\text { the use of electronic transmission } \\
\text { but there were system limitations } \\
\text { that prevented electronic } \\
\text { transmission and required a fax or } \\
\text { phoned in prescription. } \\
\text {-Inconsistent renewal processes } \\
\text { by physicians required pharmacy } \\
\text { staff to complete or edit fields }\end{array}$ & $\begin{array}{c}\text { Journal of American } \\
\text { Medical Informatics } \\
\text { Association }\end{array}$ & B \\
\hline 2011 & Liu, Burkhart, Bell & $\begin{array}{l}\text { Evaluation of the NCPDP } \\
\text { Structured and Codified Sig } \\
\text { Format for e-prescriptions }\end{array}$ & $\begin{array}{c}\text { Retrospective } \\
\text { cross-sectional study }\end{array}$ & $\begin{array}{c}\text { Evaluation of } \\
\text { ambulatory } \\
\text { e-prescriptions based on } \\
\text { the actual words used by } \\
\text { prescribers in the sig } \\
\text { field to the equivalent } \\
\text { terms in the designated } \\
\text { terminology } \\
\end{array}$ & $\begin{array}{l}\bullet 95 \% \text { of sigs were correctly } \\
\text { represented } \\
\text { - Some ambiguities - but mainly } \\
\text { from multi-step instructions } \\
\text { - Most errors were administration } \\
\text { timings and dose delivery } \\
\text { methods }\end{array}$ & $\begin{array}{l}\text { Journal of the } \\
\text { American Medical } \\
\text { Informatics } \\
\text { Association }\end{array}$ & B \\
\hline 2011 & $\begin{array}{l}\text { Nanji, Rothschild, } \\
\text { Salzberg, Keohane, } \\
\text { Zigmont, Devita, } \\
\text { Gandhi, Dalal, Bates, } \\
\text { Poon }\end{array}$ & $\begin{array}{l}\text { Errors associated with } \\
\text { outpatient computerized } \\
\text { prescribing systems }\end{array}$ & $\begin{array}{l}\text { Retrospective cohort } \\
\text { study }\end{array}$ & $\begin{array}{l}\text { Clinical panel review of } \\
3850 \text { computer } \\
\text { generated prescriptions } \\
\text { for medication errors, } \\
\text { potential adverse drug } \\
\text { events, and rate of } \\
\text { prescribing errors } \\
\end{array}$ & $\begin{array}{l}\bullet 11.7 \% \text { error rate }-35 \% \text { of those } \\
\text { errors were considered potential } \\
\text { adverse drug events. } \\
\bullet \text { Main error }- \text { omitted } \\
\text { information }\end{array}$ & $\begin{array}{l}\text { Journal of American } \\
\text { Medical Informatics } \\
\text { Association }\end{array}$ & B \\
\hline 2011 & $\begin{array}{l}\text { Redwood, Rajakumar, } \\
\text { Hodson, Coleman }\end{array}$ & $\begin{array}{l}\text { Does the implementation of } \\
\text { an electronic prescribing } \\
\text { system create unintended } \\
\text { medication errors? A study of } \\
\text { the sociotechnical context } \\
\text { through the analysis of } \\
\text { reported medication incidents }\end{array}$ & $\begin{array}{c}\text { Exploratory study of } \\
\text { survey } \\
\text { collection }\end{array}$ & $\begin{array}{l}\text { Survey results and } \\
\text { determine if } \\
\text { sociotechnical incident } \\
\text { (due to human } \\
\text { interactions with the } \\
\text { system) or } \\
\text { non-sociotechnical }\end{array}$ & $\begin{array}{l}\bullet 15 \% \text { (of } 485) \text { were } \\
\text { sociotechnical issues in the } \\
\text { hospital setting } \\
\text { - Increased incidence on Sundays } \\
\text { - Need to improve training and } \\
\text { work out unintended problems }\end{array}$ & $\begin{array}{l}\text { BMC Medical } \\
\text { Informatics and } \\
\text { Decision Making }\end{array}$ & B \\
\hline 2011 & $\begin{array}{c}\text { Saverno, Hines, } \\
\text { Warholak, Grizzle, } \\
\text { Babits, Clark, Taylor, } \\
\text { Malone }\end{array}$ & $\begin{array}{l}\text { Ability of pharmacy clinical } \\
\text { decision-support software to } \\
\text { alert users about clinically } \\
\text { important drug-drug } \\
\text { interactions }\end{array}$ & $\begin{array}{l}\text { Qualitative } \\
\text { study }\end{array}$ & $\begin{array}{l}\text { On-site visits to analyze } \\
\text { and evaluate pharmacy } \\
\text { information systems } \\
\text { using predetermined } \\
\text { patient's and orders }\end{array}$ & $\begin{array}{l}\text { - Only } 28 \% \text { of the pharmacies } \\
\text { were able to correctly identify } \\
\text { eligible interactions and } \\
\text { non-interactions. } \\
\text {-Comprehensive system updates } \\
\text { are perpetually needed. }\end{array}$ & $\begin{array}{l}\text { Journal of American } \\
\text { Medical Informatics } \\
\text { Association }\end{array}$ & B \\
\hline 2012 & Banks, Galvez & $\begin{array}{l}\text { E-Prescribing Adoption: A } \\
\text { Prescription for Progress }\end{array}$ & Analysis & None & $\begin{array}{l}\text { - Evaluation of where } \\
\text { e-prescribing is and how it is } \\
\text { helping prescribers and } \\
\text { pharmacists improve patient care } \\
\text { - Standardization by states for } \\
\text { e-prescribing }\end{array}$ & Health IT Buzz & B \\
\hline
\end{tabular}




\begin{tabular}{|c|c|c|c|c|c|c|c|}
\hline & & & & & $\begin{array}{c}\text { - North Dakota - given } \$ 6 \\
\text { million to adopt information } \\
\text { technology and e-prescribing and } \\
\text { has had a } 42 \% \text { increase in active } \\
\text { pharmacy adoption } \\
\text { - Need for investment and } \\
\text { positive environment to get } \\
\text { adoption } \\
\text { - Need to reduce barriers for } \\
\text { independent pharmacies and rural } \\
\text { areas }\end{array}$ & & \\
\hline 2012 & Salmon, Jiang & $\begin{array}{l}\text { E-prescribing: History, Issues } \\
\text { and Potentials }\end{array}$ & Review & None & $\begin{array}{c}\text { - Discusses pitfalls of } \\
\text { e-prescribing } \\
\text { - Emphasizes benefit gained } \\
\text { from adjusting dosages }\end{array}$ & $\begin{array}{l}\text { Online Journal of } \\
\text { Public Health } \\
\text { Informatics }\end{array}$ & B \\
\hline 2013 & $\begin{array}{l}\text { Lander, Klepser, } \\
\text { Cochran, Lomelin, } \\
\text { Morien }\end{array}$ & $\begin{array}{l}\text { Barriers to electronic } \\
\text { prescribing: Nebraska } \\
\text { pharmacists' perspective }\end{array}$ & Intervention & $\begin{array}{l}\text { Interview with } \\
\text { pharmacists or pharmacy } \\
\text { managers from } \\
\text { non-participating } \\
\text { pharmacies }\end{array}$ & $\begin{array}{l}\text { - } 43 \% \text { delaying implementation } \\
\text { due to transaction fees and } \\
\text { maintenance costs } \\
\text { - Lack of demand from } \\
\text { customers and prescribers to } \\
\text { implement it due to high start-up } \\
\text { costs } \\
\text { - } 39 \% \text { didn't understand the } \\
\text { benefits of e-prescribing and } \\
\text { unsure of how to implement it }\end{array}$ & & B \\
\hline 2013 & $\begin{array}{c}\text { Marceglia, Mazzola, } \\
\text { Bonacina, Targuini, } \\
\text { Donzelli, Pinciroli }\end{array}$ & $\begin{array}{l}\text { A comprehensive } \\
\text { e-prescribing model to allow } \\
\text { representing, comparing and } \\
\text { analyzing available systems }\end{array}$ & $\begin{array}{l}\text { Literature } \\
\text { review }\end{array}$ & None & $\begin{array}{l}\text { - Mentions that there is still a } \\
\text { variety of e-prescribing systems } \\
\text { and that there is a need for } \\
\text { model-based implementation to } \\
\text { increase quality of care and } \\
\text { effectiveness of care delivery }\end{array}$ & $\begin{array}{l}\text { Methods of } \\
\text { Information in } \\
\text { Medicine }\end{array}$ & $\mathrm{C}$ \\
\hline
\end{tabular}




\section{Results}

A total of fifty-six peer-reviewed articles were included in this review.Trends show that most clinicians view e-prescribing positively despite some limitations. Limitations of e-prescribing include cost of implementation and maintenance, insufficient training, and lack of standardization.As more legal regulations come into effect promoting standardization, there is greater incentive to invest in integrated e-prescribing systems as well as promote clinician adoption.

\section{Cost Effectiveness}

Illegible prescriptions and drug interactions have been attributed to causing approximately 7,000 deaths per year [59].E-prescribing has been shown to reduce prescribing errors and increase long term benefits [1].One health system in Michigan saved \$3 million annually with the implementation of e-prescribing [59].Some of the benefits include: better legibility, less prescription duplications, and improved efficiency of processing in dispensing medication.In 2010, Kaushal and colleagues showed that error rates when converting from paper prescriptions to electronic prescriptions decreased by sevenfold [2].Furthermore, pharmacist, physician, nurse and staff efficiency has been shown to improve with e-prescribing [20].In addition, the use of electronic prescribing has been shown to increase patient medication compliance, which then further decreases the number of subsequent hospital visits [18].An example is a 2011 study that showed for every $\$ 10$ increase in a patient's initial hyperlipidemia medication, the likelihood for a patient to be at goal decreases by $5 \%$ [11].A second study showed that for every dollar invested to increase medication adherence, there is a potential to save $\$ 3-\$ 10$ in medical costs [19].For some health plans, this could be a total saving of $\$ 14$ billion/year.

While the potential savings seem to be substantial, one of the biggest reasons for the lack of implementation of e-prescribing is the cost investment. The Health Information Technology for Economic and Clinical Health (HITECH) Act of 2009 was developed to pave the way towards making a technological approach to healthcare by setting aside $\$ 22$ billion for EHR utilization [8].Once EHRs have been successfully implemented within a clinical setting, the progression is to focus on electronic prescribing.However, with the HITECH Act only focusing on EHR implementation, institutions are faced with having to invest their own funds in an e-prescribing system.In addition, there are no set standards that are required for an e-prescribing program and thus institutions and facilities have freedom in the type of system they choose. A 2007 study by Scalise and colleagues revealed that the cost to implement a basic e-prescribing program ranges from $\$ 1,500$ - $\$ 4,000$ per physician.The price for an advanced system with alerts, reminders and system integration is $\$ 29,000$ per physician in the first year and $\$ 4,000$ per physician every year thereafter [9].While there is a huge difference in pricing, multiple studies have shown that physicians will utilize the system more if it is user-friendly, integrated to have access to patient files and provides clinical and formulary decision support options $[10.11,12,13,14]$. With many different commercially systems available and a multitude of different options and features, an issue arises in choosing a system and validating the investment cost.This decision is further complicated by the need to assess costs to train employees, technological maintenance of the program and ensure that the system is reducing error and providing a return on investment (ROI).Studies show that while e-prescribing software has been shown to be cost-effective for practices of all sizes, the time frame for a return on investment varies.Larger practices achieve a quicker ROI [20].Therefore, it is not difficult to imagine that many institutions are awaiting an "all-in-one" package and delaying the need to invest in electronic prescribing [15].

Pharmacies are also hesitant to implement e-prescribing into their workplace.A recent 2013 study by Banks and Galvez assessed that pharmacies did not want to spend initial start-up costs as well as maintenance and transaction fees to insurers [27].Furthermore, the lack of demand for use from physicians or patients promotes a delay in adoption.Some pharmacies commented that they didn't understand the benefits to be gained from using e-prescribing while others reported that the costs didn't outweigh the benefits.Additionally, independent pharmacies or pharmacies in rural areas have identified lack of prescription volume as a reason not to invest in such a costly program. On the other hand, with the HITECH Act of 2009, many states are providing financial assistance to offset those costs.In Tennessee, the state's pharmacists association allocated $\$ 675,000$ in grants to 124 independent, community pharmacies [28].The state of North Dakota dispersed over $\$ 6$ million to promote the adoption of information technology and has seen a $42 \%$ increase in active pharmacy utilization [28].Other states are following suit and offering state appropriations, grants and revolving loans so that smaller pharmacies can meet their technological needs, especially with the changes in state and federal requirements advocating for e-prescribing.

Incentives for pharmacies and institutions to implement adoption can result in direct cost savings.A 2013 study by Radley et al. showed that the decrease in prescription error from e-prescribing caused a $12.5 \%$ reduction in medical errors in over 4,700 hospitals in the United States [41].This translates to cost savings for over 17 million errors per year.Utilization of e-prescribing with formulary decision support can further reduce hospital visits and pharmacy spending on medication.Findings from recent articles revealed that physicians are more likely to prescribe tier 1 (generic) drugs $[10,25,26]$ and patients are more likely to purchase the tier 1 medication prescribed when it requires lower copayment.Furthermore, the resulting decrease in utilization of tier 2 (brand name) or other specialty drugs can result in savings of $\$ 845$ per patient based on a $20 \%$ 
e-prescribing rate [13].Therefore, savings can substantially increase with a higher e-prescribing adoption percentage. Pharmacies may also achieve savings by not having to purchase specialized and expensive inventory that is either a rarely dispensed item or one that a patient rarely will purchase due to its high copayment.Stocking such unpopular items results in loss of income due to items not being able to be returned to the manufacturer or becoming expired.

\section{Clinician Adoption}

The 2013 Medicare eRx Payment Adjustment provides an incentive for physicians who serve Medicare patients to switch to e-prescribing.Physicians who do not switch to e-prescribing receive a $1.5 \%$ decrease in payment on all Medicare Part B services[5].Furthermore, this adjustment increases to $2 \%$ in 2014[5].A major component for the continuing success and development of e-prescribing is its adoption by physicians, pharmacists, and any other healthcare providers.Many studies have discussed the mixed positive and negative perceptions on adoption and how negative attitudes are preventing the utilization of e-prescribing.Recent studies have shown that physicians' perception of e-prescribing after efficient implementation is viewed as very efficient and easy to use[31,32,39].A 2007 study analyzed the time to track both physician and staff members when using an integrated system. The results showed e-prescribing had no disruption to workflow and had no statistically significant increase in time when compared to handwritten prescriptions[4,40]. The use of computerized physician order entry (CPOE) in hospitals to write inpatient prescriptions was found to decrease the likelihood of a prescription error in an acute setting by $48 \%[41]$. While cost was one of the main factors contributing to negative attitudes, there were other factors as well.Lack of adequate training, clinical support, formulary support, or software issues were identified as major deterrents[29,32,36]. With the exception of lack of training, most barriers were related to the technology.Physicians reported issues of limited connectivity with pharmacies requiring multiple transmissions of the same prescription and pharmacies reported issues in receiving the same prescription more than once[10,32].This transmission issue created an increased workload for both physicians and pharmacists.If resolution of these issues is possible in the future, most physicians will have a more positive mindset towards electronic prescription adoption.For example, physicians with an integrated e-prescribing system who had access to their patient's medication profile were more likely to review it before prescribing a new medication. The use of this feature gave the physician more awareness of potential duplication in therapy, potential allergies and drug-drug interactions[2,36].Increased awareness in this manner gives physicians a better ability to evaluate and diagnose their patients.
Studies on pharmacist's perceptions revealed that they felt e-prescribing may decrease errors and increase efficiency for physicians, but required as much pharmacist intervention as would normally be provided due to omitted information or dosing errors on prescriptions sent electronically[30,32].Additionally, some pharmacists negatively viewed e-prescribing systems due to the over utilization of alerts.On the other hand, several studies showed that pharmacists perceived e-prescribing positively noting better legibility, improved processing time and efficiency as benefits[1].

Although clinician views on the adoption of e-prescribing are mixed, there is a consensus from all groups that proper training is required to improve the efficiency of the dispensing process[29,30,32]. Clinicians may receive incomplete information from time to time which results in distrust in the e-prescribing system.For example, a physician may find incomplete documentation of a patient's medical history[26].Findings from a 2011 study by Nanji and colleagues showed that for the 45 pharmacy interventions required for 3,850 computer generated prescriptions, thirty five percent of those interventions resulted from omitted information or potential adverse drug events[17].Findings from another study in 2012 revealed that 153 pharmacy interventions were needed for 1,678 new prescriptions to address omitted information, excessive quantity, etc.[40].Greater data accuracy and completeness is needed to increase clinician confidence in e-prescribing.

Clinicians also feel that some prescriptions required a faxed or phoned in prescription, citing system limitations especially with controlled substances preventing electronic transmission[32]. As of 2010, the DEA has authorized the use of electronic prescribing of controlled substances (EPCS)[34,35].However, most pharmacies have not yet adopted the utilization and will not allow physicians to electronically prescribe them and therefore, must submit a fax or phoned in prescription[35]. While controlled substances only make up $11 \%$ of all prescriptions issued, they are prescribed by $90 \%$ of all physicians[34].This creates an increase in workflow for both physicians and pharmacies.However, the adoption of EPCS is due to the extra technology regulations to ensure secure transmission by the DEA.Many systems are still in the process of being updated to include these operational security issues.Hopefully, once in effect, the issue of controlled substances will be one less barrier for the proper utilization of e-prescribing.

In addition to studies on controlled substances, studies are being conducted to examine whether e-prescribing can help to efficiently manage patients and their disease states more so than paper prescriptions.A 2011 study by Michelis and colleagues analyzed the effects of using electronic prescriptions to better manage hyperlipidemia patients[11].Patients that were given an electronic prescription were $59 \%$ more likely to reach their LDL goals. The study revealed that the utilization of formulary decision support helped patients to obtain their low cost 
prescriptions.Similarly, a second 2009 study by Smith et al. discussed how diabetes patients benefited from e-prescribing through the reduction of errors such as correct product selection through more legible prescriptions (i.e. Novolog versus Novolin or glipizide versus glimeperide)[23].Furthermore, results revealed that clinical decision support helped physicians and pharmacists better predict drug interactions and assisted with updating dosage regimens.Other studies have also analyzed the use of electronic prescriptions for oral chemotherapy regimens[42].Authors developed a built in field for weight and body surface area to allow for more accurate dosing.Findings revealed that over six hundred clinicians readily accepted and utilized e-prescribing to better enhance oral chemotherapy safety.As illustrated, several studies have highlighted positive benefits in the use of e-prescribing, however, an equal number of studies point to its limitations.

\section{Barriers to Implementation (Limitations)}

A major concern that has prohibited the widespread adoption of e-prescribing is the lack of standardization or requirements related to it $[43,57]$.This makes it difficult to assess the value of the different systems available. There are also inconsistencies in the perception of e-prescribing. Some systems are stand-alone and used only to write prescriptions while others are integrated and allow access to patient profiles.Some systems have a combination of varying clinical decision support and formulary decision support while others have none.In 2013, Marceglia and colleagues discussed potential modeling methods to better standardize e-prescribing systems by breaking down the six phases of the e-prescribing process [57].However, they recognized that it is a difficult task due to the large heterogeneity among functionalities and performances of existing systems. There is even variation in the sig codes designated for each system on how to use short codes to type in directions for electronic prescriptions [44].The National Council for Prescription Drug Programs (NCPDP) developed a structured and codified sig format that has been shown to be $95 \%$ effective. However, not all systems and institutions utilize this format because it is merely a recommendation and not a guideline that is enforced.

The second concern about e-prescribing relates to sociotechnical error, or an error caused only by human interaction when using the system [45]. It has been suggested that institutions and pharmacies develop an auditing system to ensure staff members are using the system correctly [28].Examples of sociotechnical errors are omitting information in a certain field, inappropriate use or selection of default doses, or a system alert that causes a distraction and interruption of workflow that ultimately causes a prescription error.For example, over-alerts from e-prescribing systems have been shown to be distractions hindering pharmacists' ability to discern true drug interactions.In 2011, Saverno and colleagues analyzed pharmacy e-prescribing systems in hospital and community settings and found that only $28 \%$ were able to correctly identify eligible interactions [37].A second 2011 European study showed that $93.7 \%$ of 2,729 electronic prescriptions generated an interaction alert.Of those alerts, only 10 were shown to have a true contraindication and 551 average danger alerts with some risk of adverse outcomes [38].Therefore, pharmacy systems usability needs improvement so that pharmacists can more accurately prevent adverse drug events. These changes can drastically improve pharmacist outlook on e-prescribing.Although sociotechnical errors are a concern, findings from Redwood's 2011 study showed that out of 485 medication incidents, only $15 \%$ were attributed to e-prescribing [45]. While this may be viewed as a small amount, future efforts should aim to resolve these new issues and ensure that other issues do not arise from its use.

The third major concern related to e-prescribing is the lack of adequate training for clinicians.Many studies provide support for the implementation of a pharmacy informatics team to develop training education programs as well as mediate other technological issues, such as system maintenance and upgrades [26,37].With clinical guidelines constantly changing, systems need to be continually updated to reflect those new recommendations.In addition, the informatics team may develop upgrades to make the system more user-friendly or serve as the system liaison to prescribers.For example, some prescribers have indicated that a limitation to e-prescribing was physical access to a computer, which decreased time for patient interactions [47].However, with technology also constantly changing, concerns related to lack of training may be addressed through the development of application upgrades to use on hand-held portable devices [46]. The upgrades could be developed by a pharmacy informatics team to help minimize the physical dependence on a desktop computer and allow prescribers to access patient EHRs and write e-prescriptions in places that have an Internet connection.In 2007, Donyai and colleagues provided a discussion on an upgrade developed to allow indication of an "as needed" (PRN) prescription in a hospital system to minimize administration and prescription errors by $48.9 \%$ and improve communication between prescribers and nurses [48].The upgrade also reduced the need for pharmacy interventions by $38.3 \%$.

Lastly, not all e-prescribing systems have the capability to adjust dosages for special populations such as pediatric, elderly, or those with hepatic or renal impairments $[38,55]$. While studies have shown the potential benefits for such features, Frolich and colleagues showed that recommended dosing was not altered for renally impaired patients.Furthermore, in 2013, Johnson and colleagues reported that there was limited data to show a significant role for e-prescribing in the pediatrics community due to the lack of pediatric functionality [18].However, the study also highlighted the adoption of e-prescribing emphasizing that changes to current e-prescribing systems are needed. 


\section{Conclusion}

An expansion of e-prescribing is expected in the future.Despite limitations related mainly to cost investment and inconsistency of technological systems, many studies are emphasizing the benefits of e-prescribing, which include a decrease in prescription errors, improving efficiency and prospective cost savings as a result of utilization. Although studies revealing the cost effectiveness of e-prescribing are substantial, those effects will be seen over time after efficient training has been achieved.In one study, Bartlett and colleagues had 28 physicians undergo a two week training session using an integrated system with clinical and formulary decision support on mock patients.After timed assessments, the study showed that user ability would not be a barrier for adoption as long as there was a user-friendly system and a thorough one-on-one training with troubleshooting support for implementation issues $[9,60]$.However, it is realistically difficult to require clinicians to spend long periods of time training and to expect them to overcome the barrier of investing in such a highly integrated system. The Center for Improving Medication Management (CIMM), along with the cooperation of several physician groups such as the American Medical Association, provides a clinical guide to e-prescribing with annual updates [62].The guide helps to stress the clinical importance of e-prescribing as well as any updates to its use. While it is a good resource, it is not highly well-known and is limited to prescribers.Future efforts should include a target towards other groups involved in the process, such as nurses, physician assistants and pharmacists.

Adoption of e-prescribing can be stimulated by organizational commitment from all members of the healthcare team and technological support teams $[9,56]$.This includes support from payers that may clearly communicate the benefits of e-prescribing, such as initiating a pay-for-performance program to provide a strong incentive.E-prescribing vendors can and should also proactively reach out to clinicians to ensure increased use over time.Informatics teams should develop new applications to keep the technology up-to-date and more user-friendly.Quality management programs are needed to promote the standardization of e-prescribing.Currently, Surescripts is one company aiming to help provide information on this process with the Quality Program.Their goal is to improve the quality of the e-prescribing process by assessing all aspects and work on standards, certification, network monitoring and providing customer support [61].Surescripts works with physician groups, system vendors and pharmacies to collect data to reduce the amount of time a problem is identified and solved.However, the Surescripts' program does not include every provider group, vendor and pharmacy because there are no clear laws as to what is required for the e-prescribing process. The program needs legislative support to ensure that those involved are required to be regulated by a third party.
Positive perception of e-prescribing will be the key to increasing adoption by healthcare professionals.Many physicians and pharmacists seem to focus on the barriers as opposed to the benefits.It is important to facilitate e-prescribing adoption by targeting factors that are salient to each group of clinicians.Further investment in a pharmacy informatics team, especially within institutions, can address these issues of implementation, training, and development of the continuous technological maintenance that e-prescribing systems require.Although a valuable resource, pharmacy informatics, specifically e-prescribing is still relatively a new concept and further assessments are warranted.

\section{Acknowledgements}

We are very grateful to Mercer University and Swilley Library for allowing us access to their resources to conduct our article search.

\section{REFERENCES}

[1] M. Rupp, T. Warholak. Evaluation of e-prescribing in chain community pharmacy: best-practice recommendations, Journal of the American Pharmacists Association, Vol. 48, No.3, 364-370

[2] R. Kaushal, et al. Electronic prescribing improves medication safety in community-based office practices, Journal of General Internal Medicine, Vol. 25, No.6, 530-536.

[3] Surescripts, 2012 National Progress Report, Online available from http://www.surescripts.com/about-e-prescribing/progre ss-reports/national-progress

[4] W. Hollingowrth. The Impact of e-prescribing on Prescriber and Staff Time in Ambulatory Care Clinics: A Time-Motion Study, Journal of the American Medical Informatics Association,Vol. 14, No.6, 722-730.

[5] J. Kannry. Effect of E-Prescribing Systems on Patient Safety, Mount Sinai Journal of Medicine, Vol. 78, No.6, 827-833.

[6] Centers for Medicare and Medicaid Services, Electronic Prescribing Incentive Program, Online available from http://www.cms.gov/Medicare/Quality-Initiatives-Patient-As sessment-Instruments/ERxIncentive/index.html?redirect=/er xincentive

[7] M. McBride. E-Prescribing Prevents Errors, Medical Economics, Vol. 89, No.21, 61-64.

[8] HITECH Act Summary, Online available from http://www.hitechanswers.net/about/about-the-hitech-act-of$2009 /$.

[9] D. Scalise. The case for e-prescribing, Hospital and Health Networks, Vol. 81, No.2, 45-51.

[10] J. Grossman, et al. Physician's Experiences Using Commercial E-Prescribing Systems, Health Affairs, Vol. 26, No.3, 393-404. 
[11] K. Michelis, et al. Effect of electronic prescription on attainment of cholesterol goals, Clinical Cardiology, Vol. 34, No.4, 254-260.

[12] I. Savage, et al. Medication errors with electronic prescribing (eP): Two views of the same picture, BMC Health Services Research, Vol. 10, 135.

[13] M. Fischer, et al. Effect of electronic prescribing with formulary decision support on medication use and cost, The Journal of the American Medical Association Internal Medicine, Vol. 168, No.22, 2433-2439.

[14] J. Tanne. Electronic prescribing could save at least \$29 billion, BMJ, Vol. 328, No.7449, 1155.

[15] J. Halamka, et al. E-Prescribing collaboration in Massachusetts: early experiences from regional prescribing projects, Journal of the American Medical Informatics Association, Vol. 13, No.3, 239-244.

[16] R. Vanderbush, et al. Implementing Pharmacy Informatics in College Curricula: The AACP Technology in Pharmacy Education and Learning Special Interest Group, American Journal of Pharmaceutical Education, Vol.71, No.6, 117.

[17] K. Nanji, et al. Errors associated with outpatient computerized prescribing systems, Journal of the American Medical Informatics Association, Vol. 18, No.6, 767-773.

[18] K. Johnson, C. Lehmann. Electronic prescribing in pediatrics: toward safer and more effective medication management, Pediatrics, Vol. 131, No.4, 1350-1356.

[19] Surescripts. E-prescribing shown to improve outcomes, save billions. Study quantifies relationship between e-prescribing and medication adherence, with potential savings of $\$ 140$ billion over the next 10 years, Health Management Technology, Vol. 33, No.4, 22-23.

[20] S. Corley. Electronic prescribing: a review of costs and benefits, Topics in Health Information Management, Vol. 24, No.1, 29-38.

[21] Centers for Medicare and Medicaid Services. E-prescribing incentive program, Online available from http://www.cms.gov/Medicare/E-Health/Eprescribing/index. html.

[22] D. Gaylin, et al. Public Attitudes about Health Information Technology and its Relationship to Health Care Quality, Costs, and Privacy, Health Services Research, Vol. 46, No.3, 920-938.

[23] M. Smith, D. Dang, J. Lee. E-Prescribing: Clinical Implications for Patients with Diabetes, Journal of Diabetes Science and Technology, Vol. 3, No.5, 1215-1218.

[24] U.S. Department of Health and Human Services. E-Prescribing Adoption: A Prescription for Progress, Online available from http://www.healthit.gov/buzz-blog/state-hie/e prescribing-adoption/.

[25] C. Tseng, et al. Health information technology and physician's knowledge of drug costs, The American Journal of Managed Care, Vol. 16, No.4, 105-110.

[26] J. Crosson, et al. Early adopters of electronic prescribing struggle to make meaningful use of formulary checks and medication history documentation, Journal of the American Board of Family Medicine, Vol. 25, No.1,24-32.
[27] L. Lander, et al. Barriers to Electronic Prescribing: Nebraska Pharmacists' Perspective, The Journal of Rural Health, Vol. 29, No.1, 24-32.

[28] U.S. Department of Health and Human Services. E-Prescribing Adoption: A Prescription for Progress, Online available from http://www.healthit.gov/buzz-blog/state-hie/e prescribing-adoption/.

[29] O. Odukoya, M. Chui. Relationship between e-prescriptions and community pharmacy workflow, Journal of the American Pharmacists Association, Vol. 52, No. 6, 168-174.

[30] T. Warholak, M. Rupp. Analysis of community chain pharmacists' interventions on electronic prescriptions, Journal of the American Pharmacists Association, Vol. 49, No. 1, 59-64.

[31] K. Lapane, R. Rosen, C. Dube. Perceptions of e-prescribing efficiencies and inefficiencies in ambulatory care, International Journal of Medical Information, Vol. 81, No.1, 39-46.

[32] J. Grossman, et al. Transmitting and processing electronic prescriptions: experiences of physician practices and pharmacies, Journal of the American Medical Informatics Association, Vol. 19, No.3, 353-359.

[33] J. Ancker, et al. Consumer experience with and attitudes toward health information technology: a nationwide survey,Journal of the American Medical Informatics Association, Vol. 20, No.1, 152-156.

[34] C. Thomas, et al. Prescribers' expectations and barriers to electronic prescribing of controlled substances, Journal of the American Medical Informatics Association, Vol. 19, No.3, 375-381.

[35] C. Thomas, et al. Early experience with electronic prescribing of controlled substances in a community setting, Journal of the American Medical Informatics Association, Vol. 20, No.1, 44-51.

[36] C. Desroches, et al. Differences between integrated and stand-alone E-prescribing systems have implications for future use, Health Affairs (Millwood), Vol. 29, No.12, 2268-2277.

[37] K. Saverno, et al. Ability of pharmacy clinical decision-support software to alert users about clinically important drug-drug interactions, Journal of the American Medical Informatics Association, Vol. 18, No.1, 32-37.

[38] T. Frolich, et al. Evaluation of medication safety in the discharge medication of 509 surgical inpatients using electronic prescription support software and an extended operational interaction classification, European Journal of Clinical Pharmacology, Vol. 67, No.12, 1273-1282.

[39] J. Spina, et al. Potential safety gaps in order entry and automated drug alerts: a nationwide survey of VA physician self-reported practices with computerized order entry, Medical Care, Vol. 49, No.10, 904-910.

[40] A. Gilligan, et al. Analysis of pharmacists' interventions on electronic versus traditional prescriptions in 2 community pharmacies, Research in Social and Administrative Pharmacy, Vol. 8, No.6,523-532.

[41] D. Radley, et al. Reduction in medication errors in hospitals due to adoption of computerized provider order entry 
systems, Journal of the American Medical Informatics Association, Vol. 20, No.3, 470-476.

[42] S. Weingart, et al. Improving electronic oral chemotherapy prescription: can we build a safer system?" Journal of Oncology Practice, Vol. 8, No.6, 168-173.

[43] S. McMullin. Standardization is necessary in the methods to assess the value of electronic prescribing systems, Journal of Managed Care Pharmacy, Vol. 11, No.7, 594-595.

[44] H. Liu, Q. Burkhart, D. Bell. Evaluation of the NCPDP Structured and Codified Sig Format for e-prescriptions, Journal of the American Medical Informatics Association,Vol. 18, No.5, 645-651.

[45] S. Redwood, et al. Does the implementation of an electronic prescribing system create unintended medication errors?A study of the sociotechnical context through the analysis of medication incidents, BMC Medical Informatics and Decision Making, Vol. 11, No.29.

[46] S. Fischer, et al. Handheld computing in medicine, Journal of the American Medical Informatics Association, Vol. 10, No.2, 139-149.

[47] K. Galt, et al. Physician use of hand-held computers for drug information and prescribing, IN Advances in Patient Safety. Volume 4, Agency for Healthcare Research and Quality,Rockville, 2005.

[48] P. Donyai, et al. The effects of electronic prescribing on the quality of prescribing, British Journal of Clinical Pharmacology, Vol. 65, No.2, 230-237.

[49] G. Schiff, T. Rucker. Computerized prescribing: building the electronic infrastructure for better medication usage, The Journal of the American Medical Association, Vol. 279, No.13, 1024-1029.

[50] J. Overhage, et al. A randomized trial of 'corollary orders' to prevent errors of omission, Journal of the American Medical Informatics Association, Vol. 4, No.5,364-375.

[51] D. Bates, et al. Effect of computerized physician order entry and a team intervention on prevention of serious medication errors, The Journal of American Medical Association, Vol. 280, No.15, 1311-1316.
[52] D. Bates, et al. The impact of computerized physician order entry on medication error prevention, Journal of the American Medical Informatics Association, Vol. 6, No.4, 313-321.

[53] A. Potts, et al. Computerized physician order entry and medication errors in a pediatric critical care unit, Pediatrics, Vol. 113, No. 1, 59-63.

[54] G. Chertow, et al. Guided medication dosing for inpatients with renal insufficiency, Journal of the American Medical Association, Vol. 286, No.22, 2839-2844.

[55] J. Salmon, R. Jiang. E-prescribing: History, Issues and Potentials, Online Journal of Health Informatics, Vol. 4, No.3.

[56] L. Pizzi, et al. Factors related to physicians' adoption of electronic prescribing: results from a national survey, American Journal of Medical Quality, Vol. 20, No.1, 22-32.

[57] S. Marceglia, et al. A comprehensive e-prescribing model to allow representing, comparing and analyzing available systems, Methods of Information in Medicine, Vol. 52, No.3, 199-219.

[58] C. Woodward. Digital Rx: Obama pushing for paperless prescriptions, Canadian Medical Association Journal, Vol. 180, No.8, 806.

[59] R. Vesely. The riches of e-prescribing.Insurers will say, do pretty much anything to get physicians to prescribe medications electronically. Ever wonder why? Modern Healthcare, Vol. 38, No.7, 6-7.

[60] G. Bartlett, et al. Evaluation of standardized tasks for primary care physicians using the MOXXI electronic prescribing and integrated drug management system, American Medical Informatics Association Annual Symposium, Marriott Wardman Park Hotel, 8-12, 2003.

[61] Surescripts Quality Program, Online available from http://www.surescripts.com/about-us/quality-program.

[62] The Center for Improving Medication Management. Clinician's Guide to e-Prescribing, Online available from http://www.ama-assn.org//resources/doc/hit/clinicians-guideerx.pdf. 